\title{
Accounting for Environmental Variability, Modeling Errors, and Parameter Estimation Uncertainties in Structural Identification
}

\author{
Iman Behmanesh and Babak Moaveni \\ Department of Civil and Environmental Engineering, Tufts University, Medford, MA, USA
}

\begin{abstract}
This paper presents a Hierarchical Bayesian model updating framework to account for the effects of ambient temperature and excitation amplitude. The proposed approach is applied for model calibration, response prediction and damage identification of a footbridge under changing environmental/ambient conditions. The concrete Young's modulus of the footbridge deck is the considered updating structural parameter with its mean and variance modeled as functions of temperature and excitation amplitude. The identified modal parameters over 27 months of continuous monitoring of the footbridge are used to calibrate the updating parameters. One of the objectives of this study is to show that by increasing the levels of information in the updating process, the posterior variation of the updating structural parameter (concrete Young's modulus) is reduced. To this end, the calibration is performed at three information levels using (1) the identified modal parameters, (2) modal parameters and ambient temperatures, and (3) modal parameters, ambient temperatures, and excitation amplitudes. The calibrated model is then validated by comparing the model-predicted natural frequencies and those identified from measured data after deliberate change to the structural mass. It is shown that accounting for modeling error uncertainties is crucial for reliable response prediction, and accounting only the estimated variability of the updating structural parameter is not sufficient for accurate response predictions. Finally, the calibrated model is used for damage identification of the footbridge.
\end{abstract}

Keywords: Hierarchical Bayesian model updating, Effects of changing environmental conditions, modeling errors, response prediction, damage identification

\section{Introduction}

Finite element (FE) Model updating is the process of calibrating model parameters to minimize the difference between the model-calculated and measured structural responses. The updated FE models provide a better understanding of the structural behavior and can be used for structural health and performance assessment, and response prediction under future loading events [1]. A common type of experimental data in practice is vibration measurements. In the case of linear model updating applications, 
the misfits between dynamic features (e.g., modal parameters) extracted from the measurements and those predicted by the model are considered as residuals to be minimized. Some applications of deterministic and probabilistic FE model updating methods for model calibration and damage identification of civil structures using identified modal parameters can be found in [2-7] and [8-11], respectively. Bayesian FE model updating framework has been developed for model calibration [12-14], damage identification [9, 15-17], model class selection [18, 19], and reliability analysis [20, 21] since its introduction by Beck and Katafygiotis in [22]. A review on the application of Bayesian frameworks for structural identification can be found in [23-25]. In this framework, the prior probability distributions of the model parameters are updated to their posterior probability distributions given the measured data. Recently, the Hierarchical Bayesian updating framework was introduced by the authors in [26] that can be used for identification of structural systems with inherent variability in their mass and/or stiffness properties due to changing environmental and ambient conditions. In structural identification applications, the environmental and ambient conditions are complementary information to the identified modal parameters and accounting for their effects on the structural properties (mass and/or stiffness) can result in more accurate identifications.

It is worth mentioning that the term "inherent variability" of the structural parameters does not refer to inherent randomness but to the variation of effective structural properties due to changing environmental and ambient conditions. In Bayesian framework, the probability distributions represent the state of our knowledge and not the inherent randomness of a parameter [24, 27, 28]. Therefore, given the classical categorization of uncertainties as aleatory and epistemic [29], uncertainties in Bayesian framework are only categorized as epistemic [25, 30, 31] (i.e., reducible) since our state of knowledge about a parameter can be improved by using more data/information.

The effects of changing environmental conditions on modal parameters and model updating results are well documented in the literature. Peeters and De Roeck [32] used an auto-regressive model to account the effects of temperature on natural frequencies of the Z24 Bridge. Principle Component Analysis is used in [33-35] to decouple the effects of temperature from damage on natural frequencies. However, those studies focused only on detecting the existence of damage and not on identifying the location and severity of the damage. Giraldo et al. [36], used both Principle Component Analysis and FE model updating to estimate the loss of structural stiffness due to damage under changing environmental conditions. In [37], the temperature variations are used as forcing functions for updating the structural FE model based on the strain and displacement measurements. Yuen and Kuok [38] proposed a Bayesian regression model to represent the environmental effects on a 22-story residential buildings. In [39-42], the variability of natural frequencies due to the changing environmental/ambient conditions are modeled and removed from the identified natural frequencies. The trend-removed natural frequencies can then be used for damage identification [42]. However, the calibrated models using temperature-removed data cannot be directly used for response predictions under future load events. 
In this paper, the Hierarchical Bayesian framework of [26] is extended to explicitly account for ambient temperature and excitation amplitude and their modeling uncertainties. The proposed framework is then implemented for model calibration, response prediction, and damage identification of the Dowling Hall Footbridge. The initial FE model of the footbridge is calibrated using the continuously measured vibration and temperature data over a period of 27 months. Effects of temperature and excitation amplitude are modeled on the effective Young's modulus of bridge deck in the Hierarchical framework. The structural model is calibrated given three information levels, and the prediction uncertainties of the concrete Young's modulus from the three information levels are compared. These levels include: (1) the identified modal parameters only, (2) the identified modal parameters and the ambient temperature measurements, and (3) the identified modal parameters, the temperature measurements and a measure of the excitation levels during each test. The importance of propagating model uncertainties in response prediction applications is discussed. The proposed technique is also used for identifying simulated damage of the footbridge under changing environmental conditions.

The completed study is presented in the following sections. In Section 2, the Dowling Hall Footbridge and its continuous monitoring system is briefly introduced. Section 3 briefly reviews the Hierarchical Bayesian model updating framework. Section 4 presents the application of the Hierarchical Bayesian updating framework for model calibration of the footbridge based on different levels of information. The calibrated FE model is then used for response prediction in Section 5 and damage identification in Section 6. Finally, Section 7 provides the concluding remarks.

\section{Dowling Hall Footbridge}

\subsection{Instrumentation}

Dowling Hall footbridge is located at Tufts University, Medford campus. Error! Reference source not found. shows the south view of the footbridge, which is $3.9 \mathrm{~m}$ wide and consists of two $22 \mathrm{~m}$ spans. The footbridge is composed of a reinforced concrete deck and a steel frame. In the fall 2009, eight accelerometers and ten thermocouples were installed on the footbridge. Error! Reference source not found. shows the location of the accelerometers. In the summer 2013, four accelerometers were added to

the existing 8-sensors monitoring setup. The locations of the newly-added accelerometers are shown by A9-A12. The monitoring system records five minutes of the bridge acceleration response every hour. More information about the footbridge and its monitoring system can be found in [43]. 


\subsection{System identification}

Stochastic Subspace Identification method [44] is used to extract the modal parameters from acceleration time histories. In our previous works [39, 41, 45], we identified and used the first 6 vibration modes; these modes are shown in Error! Reference source not found.. Based on the system identification results of a 12-sensor setup, we detected two closely-spaced modes with very similar mode shapes, one around $5.93 \mathrm{~Hz}$ (combined vertical-torsional mode) and one around $6.10 \mathrm{~Hz}$ (pure bending vertical mode). The mode shapes of the two modes could not be distinguished in the 8-sensor setup because their difference is at the locations that were not instrumented. Also, the mode at $6.10 \mathrm{~Hz}$ is difficult to be identified in the 8 -sensor configuration and is associated with relatively high estimation uncertainties. To mitigate the effects of system identification errors, we exclude these two modes in our FE model updating process. Therefore, only modes 1, 3, 4, 5, and 6 in Error! Reference source not found., are used in this study. Also, a data set is excluded if either of the modes 1, 3, or 4 is not identified. Overall, 8721 sets of data are available from January 2010 to March 2012 from the 8-sensor monitoring setup with 1824 sets corresponding to temperature below the freezing point.

The effects of changing ambient temperature on natural frequencies of the footbridge were studied in [39]. It was observed that the frequencies increase drastically at temperatures below the freezing point. Error! Reference source not found. shows the identified frequencies of the first bending and the first torsional modes versus the recorded air temperatures. Similar trend was also observed for the Z24 bridge [32] and the authors suggested that the trend is due to changes in the material properties of the asphalt when temperature goes below the freezing point. For the Dowling Hall footbridge, this can be attributed to the freeze of the moist inside concrete. The identified natural frequencies are also sensitive to the level of excitations. Error! Reference source not found. also shows the identified frequencies (recorded at temperature above $5^{\mathrm{oC}}$ ) versus the root-mean-square (RMS) of the signal recorded by Sensor 3 (see Error! Reference source not found.). The trend can be due to the reduction of effective concrete stiffness at higher excitation amplitudes.

\subsection{FE model}

A linear FE model of the footbridge is created using SAP2000 [46] and MATLAB-based [47] structural analysis software FEDEASLab [48]. This model consists of 461 nodes, 406 frame elements, and 324 shell elements. The connections between all the elements are modeled as fixed. The support nodes at the campus (west) side of the bridge are considered as pins while the joints at mid-span support are modeled as rollers on springs, i.e., free to rotate in all direction, free to move in the horizontal direction, but fixed in the transversal direction, and constrained by a spring (with stiffness of 15124 $\mathrm{kN} / \mathrm{mm}$ ) in the vertical direction. The spring stiffness was estimated separately through modeling of the 
support pier at mid-span. Finally, at the east side of the bridge the support nodes are free to rotate in all directions, fixed in the horizontal and transversal directions, but constrained by a spring (stiffness of 225 $\mathrm{kN} / \mathrm{mm}$ ) in the vertical direction. Table 1 presents the natural frequencies of the initial model, the average of identified natural frequencies, and the MAC values between the model-calculated and the average of identified mode shapes. In Table 1 only modal parameters that are extracted at warm temperatures are used. To reduce the computational time in the model updating process, the nodal masses are set to zero in horizontal and transversal directions.

Table 1: Model parameters of the initial FE model and identified model parameters

\begin{tabular}{c|ccccc} 
Mode & 1 & 3 & 4 & 5 & 6 \\
\hline FE model & 4.66 & 7.05 & 8.77 & 13.01 & 13.65 \\
Identified & 4.65 & 7.08 & 8.89 & 13.11 & 13.57 \\
MAC (\%) & 99.5 & 99.4 & 99.9 & 97.3 & 98.7
\end{tabular}

\section{Review of Hierarchical Bayesian model updating}

In Bayesian FE model updating, the posterior probability density function (PDF) of the updating parameters is estimated using the likelihood of observing the data and the prior knowledge. The Hierarchical Bayesian updating framework can include multi-level priors and can estimate the inherent variability of model parameters in addition to their estimation uncertainties. The multi-level priors can include hyper-priors as well as prior distributions for the updating parameters. If the probability distribution of updating stiffness parameter, $\boldsymbol{\theta}$, can be assumed as a truncated Normal distribution with the mean $\boldsymbol{\mu}_{\theta}$ and covariance $\boldsymbol{\Sigma}_{\theta}$, the posterior joint probability distribution of all the updating parameters can be written as:

$p\left(\boldsymbol{\Theta}, \boldsymbol{\mu}_{\boldsymbol{\theta}}, \boldsymbol{\Sigma}_{\boldsymbol{\theta}}, \boldsymbol{\mu}_{\mathrm{e}}, \boldsymbol{\Sigma}_{\mathbf{e}} \mid \tilde{\boldsymbol{\lambda}}, \tilde{\mathbf{\Phi}}\right) \propto \prod_{t=1}^{N_{t}} p\left(\tilde{\boldsymbol{\lambda}}_{t}, \tilde{\boldsymbol{\Phi}}_{t} \mid \boldsymbol{\theta}_{t}, \boldsymbol{\mu}_{\mathbf{e}}, \boldsymbol{\Sigma}_{\mathbf{e}}\right) p\left(\boldsymbol{\theta}_{t} \mid \boldsymbol{\mu}_{\boldsymbol{\theta}}, \boldsymbol{\Sigma}_{\boldsymbol{\theta}}\right) p\left(\boldsymbol{\mu}_{\boldsymbol{\theta}}\right) p\left(\boldsymbol{\Sigma}_{\boldsymbol{\theta}}\right) p\left(\boldsymbol{\mu}_{\mathbf{e}}\right) p\left(\boldsymbol{\Sigma}_{\mathbf{e}}\right)$

where $\boldsymbol{\Theta}=\left\{\boldsymbol{\theta}_{1}, \ldots, \boldsymbol{\theta}_{t}, \ldots, \boldsymbol{\theta}_{N_{t}}\right\}, \theta_{t}$ is the value of the structural parameter during test $t$ and $N_{t}$ is the total number of tests (or data sets). $\tilde{\lambda}$ and $\tilde{\boldsymbol{\Phi}}$ are the identified eigenvalues and normalized (to unit length) mode shapes of the structure, $\boldsymbol{\mu}_{\mathrm{e}}$ and $\boldsymbol{\Sigma}_{\mathrm{e}}$ are the prediction error mean vector and covariance matrix, respectively. The prediction error vector is defined as:

$$
\left[\begin{array}{l}
\mathbf{e}_{\lambda_{t}} \\
\mathbf{e}_{\mathbf{\Phi}_{t}}
\end{array}\right]=\mathbf{e}_{t} \sim N\left(\boldsymbol{\mu}_{\mathbf{e}}, \boldsymbol{\Sigma}_{\mathbf{e}}\right)
$$


In Eq. (2), $e_{\lambda_{t m}}=\lambda_{m}\left(\boldsymbol{\theta}_{t}\right)-\tilde{\lambda}_{t m}$ is the eigenvalue error for test $t$ and mode $m$, while $\mathbf{e}_{\boldsymbol{\Phi}_{t m}}=\tilde{\boldsymbol{\Phi}}_{t m}-a_{t m} \boldsymbol{\Phi}_{m}\left(\boldsymbol{\theta}_{t}\right)$ is the vector of mode shape errors for test $t$ and mode $m$. $\lambda(\boldsymbol{\theta})$ and $\boldsymbol{\Phi}(\boldsymbol{\theta})$ denote the model-calculated eigenvalues and mass-normalized mode shapes, and $a_{t m}$ is the mode shape scaling factor. Note that the model-calculated mode shapes $\boldsymbol{\Phi}(\boldsymbol{\theta})$ contain only the measured degrees of freedom (DOFs). The Gibbs Sampler $[49,50]$ is then used to generate samples from the conditional probability distributions of all the parameters. More information about the implemented Hierarchical Bayesian model updating can be found in [26].

\section{Model calibration}

In this section, the effective Young's modulus of concrete deck is updated using the available $N_{t}=$ 8721 sets of data collected at the undamaged state of the footbridge. The observed variability of the identified modal parameters (Error! Reference source not found.) and therefore structural stiffness and/or mass can be due to the effects of (1) ambient temperature, (2) excitation amplitude (wind speed, traffic load), (3) pedestrians weight on the structure, (4) cable noise, and (5) estimation errors in the identification of modal parameters. This paper presents a model within the Hierarchical updating framework to account for the effects of the first two items (ambient temperature and excitation amplitude). The updating structural parameter $\theta$ is defined as a modification factor for the effective Young's modulus of concrete deck, i.e., $E=\theta E_{0}$, where $E_{0}$ is the initial value considered as 23,763 $\left[\mathrm{N} / \mathrm{mm}^{2}\right]$ and $E$ is the updated value. Note that more than one structural updating parameters could be considered for this footbridge. Considering a larger number of updating parameters often improves the data fit but at the same time increases the computational cost and can potentially cause multi-modal posterior distributions which are difficult to sample [51-53]. In this study, the considered single structural updating parameter provides reasonably accurate data fit at all the information levels and keeps the computational costs reasonable.

In order to simplify the calibration process, the prediction error of Eq. (2) are considered to be zero mean and with a diagonal covariance matrix. The diagonal terms of the $\boldsymbol{\Sigma}_{\mathrm{e}}$ are defined as $\overline{\tilde{\lambda}}_{m}^{2} \sigma_{e}^{2}$ for the eigenvalue errors and $w \sigma_{e}^{2} \mathbf{I}\left(N_{s}\right)$ for the mode shape errors. $\overline{\tilde{\lambda}}_{m}$ is the mean of the identified eigenvalues for mode $m, N_{s}$ is the number of model shape component, $w$ is a weight factor, and $\mathbf{I}$ is the identity matrix. Here, a deterministic weight factor of $N_{s}$ is considered as the weight factor, so that it the eigenvalue residual and mode shape residuals of a mode have relatively similar weights. However, the weight factor can also be considered as an updating parameter as discussed in [13, 54].

In the following subsections, three cases of model updating are performed to update the initial FE model of the footbridge using different levels of information. In Subsection 4.1, only the identified modal parameters are used for model updating while in Subsection 4.2, the temperature measurements are also considered. In this case, a model is proposed to represent the effects of temperature on the updating 
structural parameter. Finally in Subsection 4.3, the identified modal parameters, temperature measurements and the excitation amplitudes (RMS of Sensor 3) are used in the model updating process. The model between stiffness and temperature used in Subsection 4.2 is extended to account for the excitation amplitude as well.

\subsection{Information level 1: modal parameters only}

Based on the mentioned simplifying assumptions for the error mean and covariance matrix, the joint posterior probability distribution of all the updating parameters at information level 1 can be written as:

$$
p\left(\boldsymbol{\Theta}, \mu_{\theta}, \sigma_{\theta}^{2}, \sigma_{e}^{2} \mid \tilde{\boldsymbol{\lambda}}, \tilde{\mathbf{\Phi}}\right) \propto \prod_{t=1}^{N_{t}} p\left(\tilde{\boldsymbol{\lambda}}_{t}, \tilde{\mathbf{\Phi}}_{t} \mid \theta_{t}, \sigma_{e}^{2}\right) p\left(\theta_{t} \mid \mu_{\theta}, \sigma_{\theta}^{2}\right) p\left(\mu_{\theta}\right) p\left(\sigma_{\theta}^{2}\right) p\left(\sigma_{e}^{2}\right)
$$

The likelihood function is defined as:

$$
p\left(\tilde{\boldsymbol{\lambda}}_{t}, \tilde{\mathbf{\Phi}}_{t} \mid \theta_{t}, \sigma_{e}^{2}\right) \propto \frac{1}{\sigma_{e}^{N_{t m}\left(N_{s}+1\right)}} e^{\frac{-J\left(\theta_{t}, \tilde{\boldsymbol{\lambda}}_{t}, \tilde{\boldsymbol{\Phi}}_{t}\right)}{2 \sigma_{e}^{2}}}
$$

where:

$$
J\left(\theta_{t}, \tilde{\lambda}_{t}, \tilde{\boldsymbol{\Phi}}_{t}\right)=\sum_{m=1}^{N_{t m}}\left\{\overline{\tilde{\lambda}}_{m}^{-2}\left(\lambda_{m}\left(\theta_{t}\right)-\tilde{\lambda}_{t m}\right)^{2}+w^{-1}\left(a_{t m} \boldsymbol{\Phi}_{m}\left(\theta_{t}\right)-\tilde{\boldsymbol{\Phi}}_{t m}\right)^{T}\left(a_{t m} \boldsymbol{\Phi}_{m}\left(\theta_{t}\right)-\tilde{\boldsymbol{\Phi}}_{t m}\right)\right\} \quad t=1: N_{t}
$$

In Eq. (5), $N_{t m}$ is the total number of identified modes during test $t$ and is always between 3 and 5 , and the mode shape scaling factor is defined as $a_{t m}=\left(\tilde{\boldsymbol{\Phi}}_{t m}^{T} \boldsymbol{\Phi}\left(\theta_{t}\right)\right) /\left(\boldsymbol{\Phi}^{T}\left(\theta_{t}\right) \boldsymbol{\Phi}\left(\theta_{t}\right)\right)$.

The graphical representation of the updating framework is shown in Error! Reference source not

found. The most common technique to solve Eq. (3) is the Gibbs Sampler, where samples are drawn from the conditional probability distribution of each parameter. This technique can be easily applied in practice if the conditional probability distributions can be categorized as one of the standard probability distributions classes such as Normal or Gamma. However, this requirement is not satisfied for the conditional probability distributions of $\theta_{t}$ :

$p\left(\theta_{t} \mid \mu_{\theta}, \sigma_{\theta}^{2}, \sigma_{e}^{2}, \tilde{\lambda}_{t}, \tilde{\mathbf{\Phi}}_{t}\right) \propto e^{\frac{-J\left(\theta_{t}, \tilde{\lambda}_{t}, \tilde{\boldsymbol{\Phi}}_{t}\right)}{2 \sigma_{e}^{2}}-\frac{\left(\theta_{t}-\mu_{\theta}\right)^{2}}{2 \sigma_{\theta}^{2}}}$

Sampling the probability distribution of Eq. (6) needs advanced sampling techniques such as Adaptive Metropolis-Hastings [55], and it can become computationally expensive for large number of 
data sets. The computational cost in sampling can be reduced by either: (1) parallel computing [53, 56] as each $\theta_{t}$ can be sampled independently, or (2) simplifying the objective function of Eq. (5). In this study, the latter is chosen; the objective function $J\left(\theta_{t}, \tilde{\boldsymbol{\lambda}}_{t}, \tilde{\boldsymbol{\Phi}}_{t}\right)$ is approximated by a second order Taylor Series expansion:

$$
J\left(\theta_{t}, \tilde{\boldsymbol{\lambda}}_{t}, \tilde{\mathbf{\Phi}}_{t}\right) \approx J\left(\hat{\theta}_{t}, \tilde{\boldsymbol{\lambda}}_{t}, \tilde{\mathbf{\Phi}}_{t}\right)+\left.\frac{\partial^{2} J\left(\theta_{t}, \tilde{\boldsymbol{\lambda}}_{t}, \tilde{\mathbf{\Phi}}_{t}\right)}{2 \partial \theta_{t}^{2}}\right|_{\hat{\theta}_{t}}\left(\theta_{t}-\hat{\theta}_{t}\right)^{2}=J\left(\hat{\theta}_{t}, \tilde{\boldsymbol{\lambda}}_{t}, \tilde{\mathbf{\Phi}}_{t}\right)+\frac{H\left(\hat{\theta}_{t}\right)}{2}\left(\theta_{t}-\hat{\theta}_{t}\right)^{2}
$$

where $\hat{\theta}_{t}$ is the optimum $\theta_{t}$ that minimizes the objective function, and $H\left(\hat{\theta}_{t}\right)$ is the Hessian of the objective function at the optimum value $\hat{\theta}_{t}$. The first order term of the Taylor Series is omitted in Eq. (7) since the gradient of the objective function at its minimum is zero. More information on this sampling technique and its relative accuracy comparing to alternative sampling procedures can be found in Chapter 19 of [57]. To estimate the Hessian matrix, the sensitivities of eigenvalues and mode shapes are calculated from Eq. (8) and Eq. (9) [58].

$$
\begin{aligned}
& \frac{\partial \lambda_{m}}{\partial \theta_{t}}=\boldsymbol{\Phi}_{m}^{T} \frac{\partial \hat{\mathbf{k}}}{\partial \theta_{t}} \boldsymbol{\Phi}_{m} \\
& \frac{\partial \boldsymbol{\Phi}_{m}}{\partial \theta_{t}}=\sum_{q=1 \neq m}^{N_{q}} \frac{\boldsymbol{\Phi}_{q} \boldsymbol{\Phi}_{q}^{T}}{\lambda_{m}-\lambda_{q}} \frac{\partial \hat{\mathbf{k}}}{\partial \theta_{t}} \boldsymbol{\Phi}_{m}
\end{aligned}
$$

where the derivative of the stiffness matrix after static condensation can be expresses as:

$$
\frac{\partial \hat{\mathbf{k}}}{\partial \theta_{t}}=\frac{\partial \mathbf{k}_{n n}}{\partial \theta_{t}}-\frac{\partial \mathbf{k}_{z n}^{T}}{\partial \theta_{t}} \mathbf{k}_{z z}^{-1} \mathbf{k}_{z n}-\mathbf{k}_{z n}^{T} \mathbf{k}_{z z}^{-1} \frac{\partial \mathbf{k}_{z n}}{\partial \theta_{t}}+\mathbf{k}_{z n}^{T} \mathbf{k}_{z z}^{-1} \frac{\partial \mathbf{k}_{z z}}{\partial \theta_{t}} \mathbf{k}_{z z}^{-1} \mathbf{k}_{z n}
$$

In Eq. (10), $z$ denotes the degrees-of-freedoms (DOFs) with zero mass and $n$ denotes the DOFs with nonzero mass assignments. Note that this simplification is similar to Laplace Asymptotic Approximation that is used in $[9,20]$. By combining Eq. (6) and Eq. (7), the conditional probability distribution of $\theta_{t}$ can be written as a Normal distribution:

$$
p\left(\theta_{t} \mid \mu_{\theta}, \sigma_{\theta}^{2}, \sigma_{e}^{2}, \tilde{\lambda}_{t}, \mathbf{\Phi}_{t}\right) \approx N\left(\frac{\hat{\theta}_{t} \sigma_{\theta}^{2}+\mu_{\theta} \sigma_{\hat{\theta}_{t}}^{2}}{\sigma_{\theta}^{2}+\sigma_{\hat{\theta}_{t}}^{2}}, \frac{\sigma_{\theta}^{2} \sigma_{\hat{\theta}_{t}}^{2}}{\sigma_{\theta}^{2}+\sigma_{\hat{\theta}_{t}}^{2}}\right)
$$

where: 


$$
\sigma_{\hat{\theta}_{t}}^{2}=2 H^{-1}\left(\hat{\theta}_{t}\right) \sigma_{e}^{2}
$$

represents the estimation uncertainty of $\hat{\theta}_{t}$ due to modeling errors and incomplete modal information. The misfit between the model and the data is propagated to other updating parameters through $\sigma_{\hat{\theta}_{t}}^{2}$. In the absence of modeling errors $\sigma_{e}^{2}=0$ and the conditional probability distribution of $\theta_{t}$ will be a Dirac Delta function at $\hat{\theta}_{t}$. The conditional distribution of Eq. (11) is also sensitive to the completeness of modal data [59]. If more modal parameters become available, different $H\left(\hat{\theta}_{t}\right), J\left(\theta_{t}, \tilde{\lambda}_{t}, \tilde{\boldsymbol{\Phi}}_{t}\right)$, and $\sigma_{e}^{2}$ will be obtained.

It can be shown that the conditional probability distribution of the model error parameter can be written as the following if a uniform prior is assumed for $\sigma_{e}^{2}[60]$ :

$$
p\left(\sigma_{e}^{-2} \mid \boldsymbol{\Theta}, \tilde{\boldsymbol{\lambda}}, \tilde{\mathbf{\Phi}}\right)=\operatorname{Gamma}\left(\frac{N_{s}+1}{2} \sum_{t=1}^{N_{t}} N_{t m}+1,2\left(\sum_{t=1}^{N_{t}} J\left(\hat{\theta}_{t}, \tilde{\boldsymbol{\lambda}}_{t}, \tilde{\boldsymbol{\Phi}}_{t}\right)+\frac{H\left(\hat{\theta}_{t}\right)}{2}\left(\theta_{t}-\hat{\theta}_{t}\right)^{2}\right)^{-1}\right)
$$

An Inverse-Gamma prior (or Inverse-Whishart if correlations are also considered as updating parameters [26]) can also be used for this variance parameter. Considering an Inverse-Gamma prior helps the convergence of the optimizations when $\boldsymbol{\mu}_{\mathbf{e}}$ is also an updating parameter, which is not the case in this study. By assuming a Uniform prior for the $\mu_{\theta}$ and a Gamma prior for $\sigma_{\theta}^{-2}, p\left(\sigma_{\theta}^{-2}\right)=\operatorname{Gamma}(\alpha, \beta)$, the conditional probability distributions of the mean and variance of the structural parameter (concrete Young's modulus) can be written as:

$$
\begin{aligned}
& p\left(\mu_{\theta} \mid \Theta, \sigma_{\theta}^{2}\right)=N\left(\frac{1}{N_{t}} \sum_{t=1}^{N_{t}} \theta_{t}, \frac{1}{N_{t}} \sigma_{\theta}^{2}\right) \\
& p\left(\sigma_{\theta}^{-2} \mid \Theta, \mu_{\theta}\right)=\text { Gamma }\left(\frac{N_{t}}{2}+\alpha,\left(\frac{1}{\beta}+\frac{1}{2} \sum_{t=1}^{N_{t}}\left(\theta_{t}-\mu_{\theta}\right)^{2}\right)^{-1}\right)
\end{aligned}
$$

1000 Gibbs samples are generated based on the conditional probability distributions in Eq. (11) and Eq. (13) to Eq. (15). The histograms of $\theta_{1}$ (test 1) and the three hyper-parameters $\left(\mu_{\theta}, \sigma=\sigma_{\theta} / \mu_{\theta}\right.$ and $\sigma_{e}$ ) are shown in Error! Reference source not found. Note that the estimation uncertainties of the hyper-parameters are very small and therefore negligible.

\subsection{Information level 2: modal parameters and ambient temperatures}


As it was observed in Error! Reference source not found., the identified natural frequencies (and therefore the stiffness) have a nonlinear relationship with ambient temperatures. To account for this relationship, the following model is considered for the mean and variance of the Young's modulus of concrete as a function of temperature.

$$
\begin{aligned}
& \mu_{\theta}\left(T_{t}\right)=Q+S \times T_{t}+R \times\left(1-\operatorname{erf}\left(\frac{T_{t}-\Upsilon}{\tau}\right)\right) \\
& \sigma_{\theta}^{2}\left(T_{t}\right)=\mu_{\theta}^{2}\left(T_{t}\right) \times \sigma^{2}
\end{aligned}
$$

where $Q, S, R, \Upsilon, \tau$, and $\sigma$ describe the stiffness-temperature model and are the new hyper-parameters in the updating framework. The first two terms in Eq. (16) represent the linear trend observed at above freezing temperature ranges. The third term captures the trend at lower temperatures (close or below freezing point) with transition temperatures $\Upsilon$, and $\tau$ characterizes the transition range. This temperature model stems from the observed trend of natural frequencies and ambient temperature shown in Error! Reference source not found.. Eq. (17) assigns higher uncertainty at lower temperatures as the $\hat{\theta}_{t}$ values have more deviation at this range. Error! Reference source not found. shows the $\hat{\theta}_{t}$ values (already calculated in Section 4.1) versus the recorded temperatures during each test, which follow a similar trend to the identified natural frequencies. In Error! Reference source not found., the identified and modelcalculated frequencies of the first bending and the first torsional modes are presented, which suggests that calibrating the considered structural parameter can capture the observed variability of the natural frequencies.

The joint posterior probability distribution of the updating parameters can be written as Eq. (18).

$$
\begin{aligned}
& p\left(\boldsymbol{\Theta}, Q, S, R, \Upsilon, \tau, \sigma^{2}, \sigma_{e}^{2} \mid \tilde{\boldsymbol{\lambda}}, \tilde{\mathbf{\Phi}}, \mathbf{T}\right) \propto \\
& \prod_{t=1}^{N_{t}} p\left(\tilde{\boldsymbol{\lambda}}_{t}, \tilde{\mathbf{\Phi}}_{t} \mid \theta_{t}, \sigma_{e}^{2}\right) p\left(\theta_{t} \mid Q, S, R, \Upsilon, \tau, \sigma^{2}, T_{t}\right) p(Q) p(S) p(R) p(\Upsilon) p(\tau) p\left(\sigma^{2}\right) p\left(\sigma_{e}^{2}\right)
\end{aligned}
$$

The prior probability of all the hyper-parameters except $\sigma_{e}$ is set as uniform distributions. The prior $p\left(\sigma^{-2}\right)$ is set as $\operatorname{Gamma}(\alpha, \beta)$, similar to Section 4.1, with $\alpha=1$ and $\beta=2$. The conditional probability distributions of $Q, S, R, \Upsilon, \tau$, and $\sigma$ converge to a Dirac Delta function if an infinite number of data sets is available. In this application and for simplicity, we assume that the number of data sets is large enough to make the variances of the conditional probability distributions negligible. The most probable values for $Q, S, R, \Upsilon$, and $\tau$ can be calculated by minimizing Eq. (19) at each step of the Gibbs Sampler. 
$-\log \left(p\left(Q, S, R, \Upsilon, \tau \mid \boldsymbol{\Theta}, \sigma^{2}, \mathbf{T}\right)\right) \propto \frac{N_{t}}{2} \log \left(\mu_{\theta}\left(T_{t}\right)\right)+\frac{1}{2 \sigma^{2}} \sum_{t=1}^{N_{t}} \frac{\left(\theta_{t}-\mu_{\theta}\left(T_{t}\right)\right)^{2}}{\left(\mu_{\theta}\left(T_{t}\right)\right)^{2}}$

The conditional probability distributions of $\boldsymbol{\Theta}, \sigma$ and $\sigma_{e}$ are identical to Eq. (11), Eq. (13), and Eq. (15), respectively, except that $\mu_{\theta}$ and $\sigma_{\theta}$ should be replaced by $\mu_{\theta}\left(T_{t}\right)$ and $\sigma$. Error! Reference source not found. shows the histogram of $\theta_{1}$ (concrete Young's modulus estimated from data set 1) and the seven considered hyper-parameters. Note that the estimation uncertainties of $Q, S, R, \Upsilon$, and $\tau$ are due to the propagation of modeling error uncertainties $\sigma_{e}$. Significant reduction can be observed in the estimated coefficient-of-variation parameter $\sigma$ by including the temperature measurements and a mathematical model to represent temperature effects on the structural properties.

\subsection{Information level 3: modal parameters, ambient temperatures, and excitation amplitudes}

In this section, the calibration process will be performed by including the effects of excitation amplitudes in addition to the identified modal parameters and the temperature measurements. The RMS of the signal recorded at a reference channel (Sensor 3) is used to represent the level of excitation. Eq. (16) is further extended to consider the effects of excitation amplitude on the expected value of concrete Young's modulus as shown in Eq. (20).

$\mu_{\theta}\left(T_{t}, \varepsilon_{t}\right)=Q+S \times T_{t}+R \times\left(1-\operatorname{erf}\left(\frac{T_{t}-\Upsilon}{\tau}\right)\right)+Y \times \log \left(\varepsilon_{t}\right)$

where $\varepsilon_{t}$ is the RMS of the Sensor 3 during test $t$ and $Y$ is the added hyper-parameter to be estimated. It is worth noting that different model forms could be used to represent the relationship between natural frequencies versus temperature and considering a different model form may slightly improve (or worsen) the fit of temperature-frequency data. However, a slight change in temperature model is not expected to have significant effects on the estimated variability of structural parameter. The posterior joint probability distribution of the updating parameters can be written as:

$$
\begin{aligned}
& p\left(\boldsymbol{\Theta}, Q, S, R, \Upsilon, \tau, Y, \sigma^{2}, \sigma_{e}^{2} \mid \tilde{\boldsymbol{\lambda}}, \tilde{\mathbf{\Phi}}, \mathbf{T}, \boldsymbol{\varepsilon}\right) \propto \\
& \prod_{t=1}^{N_{t}} p\left(\tilde{\boldsymbol{\lambda}}_{t}, \tilde{\boldsymbol{\Phi}}_{t} \mid \theta_{t}, \sigma_{e}^{2}\right) p\left(\theta_{t} \mid Q, S, R, \Upsilon, \tau, Y, \sigma^{2}, T_{t}, \varepsilon_{t}\right) p(Q) p(S) p(R) p(\Upsilon) p(\tau) p(Y) p\left(\sigma^{2}\right) p\left(\sigma_{e}^{2}\right)
\end{aligned}
$$

The graphical representation of the Hierarchical model updating framework at this information level is shown in Error! Reference source not found. Error! Reference source not found. shows the histograms of $\theta_{1}$ and all the hyper-parameters. It is worth noting that even after accounting for the effects 
of temperature and excitation amplitude, the posterior distribution of $\theta$ still has non-zero standard deviation. This variability is due to other sources of uncertainty in the data and in the structural model. Error! Reference source not found. presents the estimated $\mu_{\theta}$ and $\sigma=\sigma_{\theta} / \mu_{\theta}$ at the three information levels. The mean $\mu_{\theta}$ is shown at different temperatures and three excitation levels, namely the minimum, average, and maximum values of RMS at Sensor 3. From this figure, it can be observed that the estimated variability of concrete Young's modulus is significantly reduced when accounting for temperature effects. This variability is further reduced by another $5 \%$ (from 0.0418 to 0.0396 ) after accounting for the effects of the excitation amplitude. Table 2 reports the statistics of the hyper-parameters; these values will be used for response prediction and damage identification of the footbridge in the next two sections. From Table 2, it can be seen that the estimation uncertainties of all the parameters except $\Upsilon$ and $\tau$ are very small. These two parameters define the transition of the $\theta$ values from warm temperatures to below freezing temperatures.

Table 2: Statistics of the updating hyper-parameters.

\begin{tabular}{c|cccccccc} 
Parameter & $Q$ & $S$ & $R$ & $\Upsilon$ & $\tau$ & $Y$ & $\sigma$ & $\log \left(\sigma_{e}\right)$ \\
\hline Mean & 1.0129 & -0.0048 & 0.1977 & -1.1012 & 3.1466 & -0.0131 & 0.0396 & -4.2541 \\
$\begin{array}{c}\text { Standard } \\
\text { Deviation }\end{array}$ & 0.0030 & 0.0001 & 0.0027 & 0.0513 & 0.0861 & 0.0004 & 0.0002 & 0.0012
\end{tabular}

\section{Response prediction under future loading}

A key advantage of Bayesian model updating methods over deterministic methods is consideration of the prediction error parameters as part of the probabilistic model [24] and the capability of considering as an updating parameter $[13,24,54,61]$. In the classical Bayesian FE model updating framework $[9,10$, $13,16,62]$, the prediction errors include both the modeling errors and the observed variability of the modal parameters. In the presented work, the test-to-test variability of modal parameters is modeled by structural hyper-parameters, while modeling error uncertainties are represented by $\sigma_{e}$. In this case, both structural hyper-parameters and $\sigma_{e}$ should be considered when predicting a quantity of interest from the model. In this section, the calibrated model of the previous section is used to predict the natural frequencies of the bridge under future ambient conditions and loading scenarios. The predictions are performed in two cases to underline the importance of propagating the modeling errors. In the first case, only the overall variability of structural model parameter (concrete Young's modulus) is considered in the predicted frequencies. The second case propagates the modeling error uncertainties to the predictions in addition to the overall variability of structural parameter. Data from two loading scenarios are used for the model validation study. The two loading scenarios are shown in Error! Reference source not found.. In loading scenario I, 2.7 Tons of concrete blocks are loaded on two segments of the footbridge deck, and in 
loading scenario II, 1.35 Tons of concrete blocks are loaded on one segment. 75 and 87 sets of data are collected from loading scenarios I and II, respectively. Modal parameters identified from the measured data are compared with model-predicted values for validation of the proposed framework in both cases of uncertainty quantification and two loading scenarios.

The predicted natural frequencies of the first bending and torsional modes given the temperature and RMS of Sensor 3 at each tests are plotted in Error! Reference source not found.. The black line is the mean (most probable) natural frequencies from the model, i.e., $\bar{\mu}_{\theta}(T, \varepsilon)$ :

$\bar{\mu}_{\theta}(T, \varepsilon)=\bar{Q}+\bar{S} \times T+\bar{R} \times\left(1-\operatorname{erf}\left(\frac{T-\bar{\Upsilon}}{\bar{\tau}}\right)\right)+\bar{Y} \times \log (\varepsilon)$

where the bar-signs on top of the parameters refers to the estimated mean values reported in Table $2, T$ and $\varepsilon$ are the temperature and the excitation amplitude. The shaded area corresponds to the prediction uncertainties due to the variability of concrete Young's modulus, i.e., $\lambda\left(\overline{\boldsymbol{\mu}}_{\boldsymbol{\theta}}(\mathbf{T}, \boldsymbol{\varepsilon}) \pm 2 \bar{\sigma} \overline{\boldsymbol{\mu}}_{\boldsymbol{\theta}}(\mathbf{T}, \boldsymbol{\varepsilon})\right)(95 \%$ confidence interval for $\lambda(\theta)$ ) where $\bar{\sigma}$ is the mean of $\sigma$ in Table 2. The identified natural frequencies from ambient vibration measurements are shown by stars in Error! Reference source not found.. It can be seen that in this case of response prediction (not accounting for modeling errors), many of the identified natural frequencies fall outside the high probability region of the predicted responses.

The second case of response prediction results are shown in Error! Reference source not found.. In this case the modeling errors are also included in the uncertainties of the predicted frequencies. The shaded areas correspond to $\lambda\left(\overline{\boldsymbol{\mu}}_{\boldsymbol{\theta}}(\mathbf{T}, \boldsymbol{\varepsilon})-2 \bar{\sigma} \overline{\boldsymbol{\mu}}_{\boldsymbol{\theta}}(\mathbf{T}, \boldsymbol{\varepsilon})\right)-2 \bar{\sigma} \overline{\bar{\lambda}}$ to $\lambda\left(\overline{\boldsymbol{\mu}}_{\boldsymbol{\theta}}(\mathbf{T}, \boldsymbol{\varepsilon})+2 \bar{\sigma} \overline{\boldsymbol{\mu}}_{\boldsymbol{\theta}}(\mathbf{T}, \boldsymbol{\varepsilon})\right)+2 \overline{\sigma_{e}} \overline{\tilde{\lambda}}$. In this case, all the identified natural frequencies fall in the high probability region of response predictions. Therefore for response prediction applications and generally simulating a quantity of interest from the calibrated FE model, propagation of structural parameter uncertainties is not sufficient and the modeling error uncertainties should be always considered. The proposed Hierarchical Bayesian model updating approach provides an estimate for the modeling errors.

It is worth noting that instead of using single parameter $\left(\sigma_{e}\right)$ to represent the modeling error uncertainties, the full covariance matrix and mean vector of the error functions in Eq. (2) can be updated as it is done in [26]. This would result in more accurate propagation of modeling error uncertainties in response prediction but would drastically increase the number of updating parameters and therefore the computational cost.

\section{Damage identification}


In this section, the calibrated model of Section 4.3 is used to identify the physically simulated damage on the Dowling Hall Footbridge. The damage identification is performed based on a new set of data collected from the structure at its damaged state and at different temperatures and excitation amplitudes.

\subsection{Simulating the effects of structural damage}

Damage on the footbridge is simulated by the addition of 2.24 metric tons of concrete blocks on a small segment of the bridge deck. Error! Reference source not found. shows the added concrete blocks on the footbridge. The added mass remained on the footbridge deck for three days providing 71 sets of data at the damaged state. The effects of the added mass on the identified natural frequencies of the first bending mode and first torsional mode of the structure are shown in Error! Reference source not found.. All data are recorded in warm temperatures. The minimum, average, and maximum air temperature is recorded as 20, 28, and 36 degrees Celsius, respectively. For damage identification, the footbridge deck is divided into seven segments and the addition of mass at each segment is considered as an updating parameter $\rho$. These segments are shown in Error! Reference source not found.. A damage identification study of the footbridge under a similar damage scenario was previously performed by the authors in [45] using a FE model updating framework without considering the inherent variability of the updating structural parameter or accounting for temperature and excitation amplitude effects.

\subsection{Methodology}

Similar to response prediction results, the damage identification results can account for different sources of errors and/or uncertainties. Propagating the modeling errors, as well as estimation uncertainties and inherent variability of the structural parameter (concrete Young's modulus) into damage parameters (added mass) which are different from the structural parameter causes challenges and complexities in deriving the analytical solutions and numerical results. This section will provide the formulation for this specific damage identification problem when accounting for temperature and excitation amplitude. Note that a similar (and simpler) formulation can be derived for damage identification cases where the change in stiffness represent damage measures. Besides, if the variation of temperature or excitation amplitude is not significant, the identification can be simplified as presented in [63].

A multivariate Lognormal distribution is assumed for the updating structural mass parameters $\rho$ with hyper-parameters $\boldsymbol{\mu}_{\boldsymbol{\eta}}$ and $\boldsymbol{\Sigma}_{\boldsymbol{\eta}}: \boldsymbol{\rho} \sim \operatorname{LogN}\left(\boldsymbol{\mu}_{\boldsymbol{\eta}}, \boldsymbol{\Sigma}_{\boldsymbol{\eta}}\right)$. In other words, the probability distribution of $\boldsymbol{\eta}=\log (\boldsymbol{\rho})$ will be a multivariate Normal distribution with mean $\boldsymbol{\mu}_{\boldsymbol{\eta}}$ and covariance matrix $\boldsymbol{\Sigma}_{\boldsymbol{\eta}}$. If $N_{t}^{d}$ independent data sets are available in the damaged state (in this section $N_{t}^{d}=71$ ), the posterior joint probability distribution of the updating parameters can be written as: 


$$
p\left(\boldsymbol{\eta}_{1}: \boldsymbol{\eta}_{N_{t}^{d}}, \boldsymbol{\mu}_{\boldsymbol{\eta}}, \boldsymbol{\Sigma}_{\boldsymbol{\eta}} \mid \tilde{\lambda}^{d}, \tilde{\boldsymbol{\Phi}}^{d}, \mathbf{T}^{d}, \boldsymbol{\varepsilon}^{d}\right) \propto \prod_{t=1}^{N_{t}^{d}} p\left(\boldsymbol{\eta}_{t} \mid \boldsymbol{\mu}_{\boldsymbol{\eta}}, \boldsymbol{\Sigma}_{\boldsymbol{\eta}}, \tilde{\lambda}_{t}^{d}, \tilde{\boldsymbol{\Phi}}_{t}^{d}, T_{t}^{d}, \varepsilon_{t}^{d}\right) p\left(\boldsymbol{\mu}_{\boldsymbol{\eta}}, \boldsymbol{\Sigma}_{\boldsymbol{\eta}}\right)
$$

where the superscript $d$ is referred to the damaged state data. The model uncertainties of the calibrated model of Section 4.3 should be propagated into the conditional probability distribution of $p\left(\boldsymbol{\eta}_{t} \mid \boldsymbol{\mu}_{\boldsymbol{\eta}}, \boldsymbol{\Sigma}_{\boldsymbol{\eta}}, \tilde{\boldsymbol{\lambda}}_{t}^{d}, \tilde{\boldsymbol{\Phi}}_{t}^{d}, T_{t}^{d}, \varepsilon_{t}^{d}\right)$.

$p\left(\boldsymbol{\eta}_{t} \mid \boldsymbol{\mu}_{\boldsymbol{\eta}}, \boldsymbol{\Sigma}_{\boldsymbol{\eta}}, \tilde{\boldsymbol{\lambda}}_{t}^{d}, \tilde{\mathbf{\Phi}}_{t}^{d}, T_{t}^{d}, \varepsilon_{t}^{d}\right)=\int p\left(\boldsymbol{\eta}_{t} \mid \boldsymbol{\mu}_{\boldsymbol{\eta}}, \boldsymbol{\Sigma}_{\boldsymbol{\eta}}, \tilde{\lambda}_{t}^{d}, \tilde{\mathbf{\Phi}}_{t}^{d}, T_{t}^{d}, \varepsilon_{t}^{d}, \sigma_{e}^{2}, \theta\right)$

$p\left(\theta \mid Q, S, R, \Upsilon, \tau, Y, \sigma^{2}, T_{t}^{d}, \varepsilon_{t}^{d}\right) p\left(Q, S, R, \Upsilon, \tau, Y, \sigma^{2}, \sigma_{e}^{2}\right) d \theta d Q d S d R d \Upsilon d \tau d Y d \sigma^{2} d \sigma_{e}^{2}$

As it can be seen from Error! Reference source not found., the estimation uncertainties of all the hyperparameters except $\Upsilon$ and $\tau$ are negligible. The uncertainties of $\Upsilon$ and $\tau$ can be ignored in warm temperature ranges, because the erf term in Eq. (20) approaches zero at warm temperature ranges. Therefore, the high dimensional integration of Eq. (24) can be simplified to:

$$
p\left(\boldsymbol{\eta}_{t} \mid \boldsymbol{\mu}_{\boldsymbol{\eta}}, \boldsymbol{\Sigma}_{\boldsymbol{\eta}}, \tilde{\boldsymbol{\lambda}}_{t}^{d}, \tilde{\boldsymbol{\Phi}}_{t}^{d}, T_{t}^{d}, \varepsilon_{t}^{d}\right)=\int_{\Theta} p\left(\boldsymbol{\eta}_{t} \mid \boldsymbol{\mu}_{\mathfrak{\eta}}, \boldsymbol{\Sigma}_{\boldsymbol{\eta}}, \tilde{\boldsymbol{\lambda}}_{t}^{d}, \tilde{\boldsymbol{\Phi}}_{t}^{d}, T_{t}^{d}, \varepsilon_{t}^{d}, \theta\right) p\left(\theta \mid T_{t}^{d}, \varepsilon_{t}^{d}\right) d \theta
$$

where:

$\theta\left(T_{t}^{d}, \varepsilon_{t}^{d}\right) \sim N\left(\bar{\mu}_{\theta}\left(T_{t}^{d}, \varepsilon_{t}^{d}\right), \bar{\mu}_{\theta}^{2}\left(T_{t}^{d}, \varepsilon_{t}^{d}\right) \times \bar{\sigma}^{2}\right)$

In Eq. (25), $p\left(\theta \mid T_{t}^{d}, \varepsilon_{t}^{d}\right)$ represents the uncertainty of $\theta$ at the recorded temperature and excitation level of test $t$, and $p\left(\boldsymbol{\eta}_{t} \mid \boldsymbol{\mu}_{\eta}, \boldsymbol{\Sigma}_{\boldsymbol{\eta}}, \tilde{\lambda}_{t}^{d}, \tilde{\boldsymbol{\Phi}}_{t}^{d}, T_{t}^{d}, \varepsilon_{t}^{d}, \theta\right)$ is the posterior distribution of the damage based on a given $\theta, \boldsymbol{\mu}_{\eta}, \Sigma_{\eta}$, and the available data at test $t$. Eq. (25) is a Monte Carlo Integration problem and its calculation is not trivial. Parallel computing $[53,56]$ is needed for accurate estimation of the integral or when the hyper-parameters have large estimation uncertainties. In this study, we discretize the $p\left(\theta \mid T_{t}^{d}, \varepsilon_{t}^{d}\right)$ into seven $\theta^{k}$ values including $\left\{\bar{\mu}_{\theta}, \bar{\mu}_{\theta} \pm \bar{\mu}_{\theta} \bar{\sigma}, \bar{\mu}_{\theta} \pm 2 \bar{\mu}_{\theta} \bar{\sigma}, \bar{\mu}_{\theta} \pm 3 \bar{\mu}_{\theta} \bar{\sigma}\right\}$ as shown in Error! Reference source not found.. Note that the damage identification process will be significantly simplified by ignoring the variability of $\theta$ and using only the $\bar{\mu}_{\theta}$ in the identification process. If the conditional distribution $p\left(\boldsymbol{\eta}_{t} \mid \boldsymbol{\mu}_{\boldsymbol{\eta}}, \boldsymbol{\Sigma}_{\boldsymbol{\eta}}, \tilde{\boldsymbol{\lambda}}_{t}^{d}, \tilde{\mathbf{\Phi}}_{t}^{d}, T_{t}^{d}, \varepsilon_{t}^{d}, \theta^{k}\right)$ is assumed as a an uncorrelated multivariate Normal distribution with a mean $\overline{\boldsymbol{\eta}}_{t k}$ and a covariance $\overline{\mathbf{V}}_{t k}$, the mean and covariance of $p\left(\boldsymbol{\eta}_{t} \mid \boldsymbol{\mu}_{\boldsymbol{\eta}}, \boldsymbol{\Sigma}_{\boldsymbol{\eta}}, \tilde{\boldsymbol{\lambda}}_{t}^{d}, \tilde{\boldsymbol{\Phi}}_{t}^{d}, T_{t}^{d}, \varepsilon_{t}^{d}\right)$ in Eq. (25) can be estimates as [64, 65]:

$\overline{\boldsymbol{\eta}}_{t} \approx \sum_{k=1}^{N_{k}} \overline{\boldsymbol{\eta}}_{t k} P\left[\theta^{k} \mid T_{t}^{d}, \varepsilon_{t}^{d} \cdot\right] \quad k=1: N_{k}$ 
$\overline{\mathbf{V}}_{t} \approx \sum_{k=1}^{N_{k}} P\left[\theta^{k} \mid T_{t}^{d}, \varepsilon_{t}^{d}\right] \overline{\mathbf{V}}_{t k}+\sum_{k=1}^{N_{k}} \operatorname{diag}\left(\left(\overline{\boldsymbol{\eta}}_{t k}-\overline{\boldsymbol{\eta}}_{t}\right)\left(\overline{\mathbf{\eta}}_{t k}-\overline{\boldsymbol{\eta}}_{t}\right)^{T}\right) P\left[\theta^{k} \mid T_{t}^{d}, \varepsilon_{t}^{d}\right]$

where $N_{k}$ is the number of discretized $\theta$ values (7 in this study), and $P\left[\theta^{k} \mid T_{t}^{d}, \varepsilon_{t}^{d}\right]$ is the probability of $\theta=\theta^{k}$ assuming the discretization of Error! Reference source not found..

To estimate the means $\overline{\boldsymbol{\eta}}_{t k}$ and the covariance matrix $\overline{\mathbf{V}}_{t k}$, we first need to use the Bayes rule:

$p\left(\boldsymbol{\eta}_{t k} \mid \boldsymbol{\mu}_{\eta}, \boldsymbol{\Sigma}_{\boldsymbol{\eta}}, \tilde{\boldsymbol{\lambda}}_{t}^{d}, \tilde{\boldsymbol{\Phi}}_{t}^{d}, T_{t}^{d}, \varepsilon_{t}^{d}, \theta_{k}\right) \propto p\left(\tilde{\boldsymbol{\lambda}}_{t}^{d}, \tilde{\boldsymbol{\Phi}}_{t}^{d} \mid \boldsymbol{\eta}_{t k}, T_{t}^{d}, \varepsilon_{t}^{d}, \theta_{k}\right) p\left(\boldsymbol{\eta}_{t k} \mid \boldsymbol{\mu}_{\boldsymbol{\eta}}, \boldsymbol{\Sigma}_{\boldsymbol{\eta}}\right)$

If the likelihood can also be considered as an uncorrelated multivariate Normal distribution $p\left(\tilde{\boldsymbol{\lambda}}_{t}^{d}, \tilde{\mathbf{\Phi}}_{t}^{d} \mid \boldsymbol{\eta}_{t k}, T_{t}^{d}, \varepsilon_{t}^{d}, \theta_{k}\right) \approx N\left(\hat{\boldsymbol{\eta}}_{t k}, \hat{\mathbf{V}}_{t k}\right)$, the means $\overline{\boldsymbol{\eta}}_{t k}$ and variances $\overline{\mathbf{V}}_{t k}$ can be written as:

$\overline{\boldsymbol{\eta}}_{t k}=\left(\hat{\mathbf{V}}_{t k}^{-1}+\boldsymbol{\Sigma}_{\boldsymbol{\eta}}^{-1}\right)^{-1}\left(\boldsymbol{\Sigma}_{\boldsymbol{\eta}}^{-1} \hat{\boldsymbol{\eta}}_{t k}+\hat{\mathbf{V}}_{t k}^{-1} \boldsymbol{\mu}_{\boldsymbol{\eta}}\right)$

$\overline{\mathbf{V}}_{t k}=\left(\hat{\mathbf{V}}_{t k}^{-1}+\boldsymbol{\Sigma}_{\boldsymbol{\eta}}^{-1}\right)^{-1}$

The described procedure can be considered as a Hierarchical Bayesian model averaging process. The mentioned simplifying assumptions allow us to rewrite the posterior joint probability distribution of Eq. (23) as Eq. (32).

$p\left(\boldsymbol{\eta}_{1}: \boldsymbol{\eta}_{N_{t}^{d}}, \boldsymbol{\mu}_{\boldsymbol{\eta}}, \boldsymbol{\Sigma}_{\boldsymbol{\eta}} \mid \tilde{\lambda}^{d}, \tilde{\mathbf{\Phi}}^{d}, \mathbf{T}^{d}, \boldsymbol{\varepsilon}^{d}\right) \propto \prod_{t=1}^{N_{t}^{d}} N\left(\boldsymbol{\eta}_{t} \mid \overline{\boldsymbol{\eta}}_{t}, \overline{\mathbf{V}}_{t}\right) p\left(\boldsymbol{\mu}_{\boldsymbol{\eta}}, \boldsymbol{\Sigma}_{\boldsymbol{\eta}}\right)$

The Gibbs Sampler can be used to sample Eq. (32). The only remained parameters to be estimated are $\hat{\boldsymbol{\eta}}_{t k}$ and $\hat{\mathbf{V}}_{t k}$. The former is the maximum likelihood estimator and can be obtained by minimizing $-\log \left(p\left(\tilde{\boldsymbol{\lambda}}_{t}^{d}, \tilde{\boldsymbol{\Phi}}_{t}^{d} \mid \boldsymbol{\eta}_{t k}, T_{t}^{d}, \varepsilon_{t}^{d}, \theta^{k}\right)\right)$. The latter is estimated from sampling the likelihood function using the adaptive Metropolis-Hastings algorithm for each data separately [55]. The likelihood function $p\left(\tilde{\lambda}_{t}^{d}, \tilde{\mathbf{\Phi}}_{t}^{d} \mid \boldsymbol{\eta}_{t k}, T_{t}^{d}, \varepsilon_{t}^{d}, \theta_{k}\right)$ is defined as the difference (between model and data) of changes in modal parameters from a reference state to the current state at a given temperature and RMS. The eigenvalue and mode shape error functions of mode $m$ are defined as [59]:

$$
\begin{aligned}
& \lambda_{m}\left(\boldsymbol{\eta}_{t k}, \theta^{k}\right)-\tilde{\lambda}_{t m}^{d} \frac{\lambda_{m}^{r}\left(\theta^{k}\right)}{\overline{\bar{\lambda}}_{m}^{r}\left(T_{t}^{d}, \varepsilon_{t}^{d}\right)}=e_{\lambda_{t m}}\left(\boldsymbol{\eta}_{t k}, \theta^{k}, T_{t}^{d}, \varepsilon_{t}^{d}\right) \sim N\left(0, \overline{\tilde{\lambda}}_{m}^{r} \sigma_{e}^{2}\right) \\
& \operatorname{MAC}_{m}^{r}\left(\theta^{k}\right)-M A C_{t m}\left(\boldsymbol{\eta}_{t k}, \theta^{k}\right)=e_{\boldsymbol{\Phi}_{t m}}\left(\boldsymbol{\eta}_{t k}, \theta^{k}\right) \sim N\left(0, w \sigma_{e}^{2}\right)
\end{aligned}
$$


where the superscript $r$ denotes the reference/undamaged state, $\lambda_{m}^{r}\left(\theta^{k}\right)$ is the model-calculated eigenvalue at the undamaged state, $\overline{\tilde{\lambda}}_{m}^{r}\left(T_{t}^{d}, \varepsilon_{t}^{d}\right)$ is the most probable identified eigenvalues at the undamaged state at temperature $T_{t}^{d}$ and excitation level $\varepsilon_{t}^{d}$, and $M A C_{m}^{r}$ is the modal assurance criterion between the average of identified and model-calculated mode shapes of mode $m$ at the undamaged condition. The $M A C_{t m}\left(\boldsymbol{\eta}_{t k}, \theta^{k}\right)$ is defined as:

$\operatorname{MAC}_{t m}\left(\boldsymbol{\eta}_{t k}, \theta^{k}\right)=\frac{\left(\left(\tilde{\boldsymbol{\Phi}}_{t m}^{d}\right)^{T} \boldsymbol{\Phi}_{m}\left(\boldsymbol{\eta}_{t k}, \theta^{k}\right)\right)^{2}}{\left\|\tilde{\boldsymbol{\Phi}}_{t m}^{d}\right\|^{2}\left\|\boldsymbol{\Phi}_{m}\left(\boldsymbol{\eta}_{t k}, \theta^{k}\right)\right\|^{2}}$

The model error parameter $\sigma_{e}$ is taken as the value estimated in Section 4.3. If the error functions are assumed uncorrelated, the negative Log-likelihood function can be written as:

$-\log \left(p\left(\tilde{\boldsymbol{\lambda}}_{t}, \tilde{\mathbf{\Phi}}_{t} \mid \boldsymbol{\eta}_{t k}, \theta^{k}, T_{t}^{d}, \varepsilon_{t}^{d}\right)\right)=\sum_{m=1}^{N_{m}}\left[e_{\lambda_{t m}}^{2}\left(\boldsymbol{\eta}_{t k}, \theta^{k}, T_{t}^{d}, \varepsilon_{t}^{d}\right)+w^{-1} e_{\mathbf{\Phi}_{t m}}^{2}\left(\boldsymbol{\eta}_{t k}, \theta^{k}\right)\right]+c$

where $c$ is a constant which will not affect the optimization results.

\subsection{Results}

The Gibbs Sampler is used to generate 5000 samples from the posterior probability distribution of Eq. (32). Error! Reference source not found. shows the probability of damage (added mass) exceedance on segment 2, and Error! Reference source not found. shows the probabilities of exceedance of the added mass for the other six segments. The colored/shaded region corresponds to the parameter estimation uncertainties of $\boldsymbol{\mu}_{\eta}$ and $\boldsymbol{\Sigma}_{\eta}$ from the Gibbs samples. The most probable added mass (darkest point along the $50 \%$ probability line shown by a star in Error! Reference source not found.) is 2.61 Tons, which is higher than the true values of 2.24 . However, the true value is still in the high probability region of the posterior.

To complete this study, overall $8721+504$ global optimization runs are performed (estimating $\hat{\theta}_{t}$ in Section $4.1+\hat{\boldsymbol{\eta}}_{t k}$ in Section 6.2), $3 \times 1000+5000$ Gibbs samples are generated (Sections 4.1-4.3 + Section 6.2), and $71 \times 1000$ samples are generated from Adaptive Metropolis-Hastings algorithm (to estimate $\hat{\mathbf{V}}_{k t}$ values in Section 6.2). More accurate results can be obtained by developing an efficient Metropoliswithin-Gibbs Sampling technique for structural identification applications. It is worth noting that the proposed updating framework comes with a high computational cost. Therefore, several simplifying assumptions are considered in the presented work. Some of these essential simplifications include updating a single structural parameter, using Taylor series expansion of Equation (7) instead of sampling 
the conditional PDF of Equation (6), excluding error bias and correlations of Equation (2), or discretization of probability distribution of Figure (19). More accurate results can be obtained by developing an efficient Metropolis-within-Gibbs sampling technique for structural identification applications especially if implemented in parallel computing form. The references $[53,56]$ for example, showed the benefit of using parallel computing in Bayesian frameworks. Those sampling techniques could not be directly used in this application as no hyper-parameters were presented in those references. However, they can be easily extended to be used in applications with hyper-parameters or generally Hierarchical Bayesian updating frameworks.

\section{Conclusions}

A Hierarchical Bayesian model updating framework is proposed to accounts for the effects of ambient temperature and excitation level on the updating structural parameters. The framework is applied for model calibration of a footbridge under severe environmental effects using the continuous health monitoring data recorded over 27 months. Three major sources of uncertainties in structural identification applications are discussed in this paper, namely (1) parameter estimation uncertainties, (2) inherent variability of structural parameters due to changing ambient/environmental conditions, and (3) modeling error uncertainties. In this paper, it is shown that the parameter estimation uncertainties are negligible if a sufficient number of data is available, and therefore, it can be considered as the least problematic source of uncertainty among the three mentioned sources. On the other hand, the variability of structural parameters due to changing environmental/ambient conditions can be significant. It is shown that by adding the temperature measurements and excitation amplitudes in an underlying model to explicitly consider their effects, the estimated inherent variability of the structural parameters are significantly reduced. The effects of modeling error uncertainties are highlighted for response prediction applications. It is shown that the variability of structural parameters alone cannot provide a realistic confidence interval for the predicted natural frequencies of the footbridge. However, by including the modeling error uncertainties all the identified natural frequencies fall in the high probability region of the modelcalculated predictions.

\section{Acknowledgement}

The authors would like to acknowledge the support of this study by the NSF Grant no. 1125624 and 1430180. The authors also acknowledge Professor Costas Papadimitriou from University of Thessaly and Professor Geert Lombaert from K.U. Leuven for their valuable feedback on this research and Ms. Alyssa 
Kody for the design of the bridge load test. The opinions, findings, and conclusions expressed in the paper are those of the authors and do not necessarily reflect the views of the individuals and organizations involved in this project.

\section{References}

[1] D.J. Inman, C.R. Farrar, V. Lopes Jr, V. Steffen Jr, Damage prognosis, Wiley Online Library, 2005.

[2] C. Farhat, F.M. Hemez, Updating finite element dynamic models using an element-by-element sensitivity methodology, AIAA journal, 31 (1993) 1702-1711.

[3] M.I. Friswell, J.E. Mottershead, Finite element model updating in structural dynamics, Kluwer Academic Publishers, Boston; Dordrecht, 1995.

[4] M. Sanayei, J.A.S. McClain, S. Wadia-Fascetti, E.M. Santini, Parameter estimation incorporating modal data and boundary conditions, Journal of Structural Engineering-Asce, 125 (1999) 1048-1055.

[5] A. Teughels, G. De Roeck, Damage detection and parameter identification by finite element model updating, Archives of Computational Methods in Engineering, 12 (2005) 123-164.

[6] S. Živanović, A. Pavic, P. Reynolds, Finite element modelling and updating of a lively footbridge: The complete process, Journal of Sound and Vibration, 301 (2007) 126-145.

[7] B. Moaveni, A. Stavridis, G. Lombaert, J.P. Conte, P.B. Shing, Finite-Element Model Updating for Assessment of Progressive Damage in a 3-Story Infilled RC Frame, Journal of Structural Engineering, 139 (2013) 1665-1674.

[8] J.D. Collins, G.C. Hart, T. Haselman, B. Kennedy, Statistical identification of structures, AIAA journal, 12 (1974) 185-190.

[9] M.W. Vanik, J.L. Beck, S.K. Au, Bayesian probabilistic approach to structural health monitoring, Journal of Engineering Mechanics-Asce, 126 (2000) 738-745.

[10] H. Sohn, K.H. Law, A Bayesian probabilistic approach for structure damage detection, Earthquake Engineering \& Structural Dynamics, 26 (1997) 1259-1281.

[11] C. Papadimitriou, D.C. Papadioti, Component mode synthesis techniques for finite element model updating, Computers \& Structures, 126 (2013) 15-28.

[12] K.V. Yuen, J.L. Beck, L.S. Katafygiotis, Efficient model updating and health monitoring methodology using incomplete modal data without mode matching, Structural Control \& Health Monitoring, 13 (2006) 91-107.

[13] K. Christodoulou, E. Ntotsios, C. Papadimitriou, P. Panetsos, Structural model updating and prediction variability using Pareto optimal models, Comput. Meth. Appl. Mech. Eng., 198 (2008) 138-149.

[14] H. Lam, H. Peng, S. Au, Development of a practical algorithm for Bayesian model updating of a coupled slab system utilizing field test data, Engineering Structures, 79 (2014) 182-194.

[15] J. Ching, J.L. Beck, Bayesian analysis of the Phase II IASC-ASCE structural health monitoring experimental benchmark data, Journal of Engineering Mechanics-ASCE, 130 (2004) 1233-1244.

[16] K.V. Yuen, J.L. Beck, S.K. Au, Structural damage detection and assessment by adaptive Markov chain Monte Carlo simulation, Struct. Control. Health Monit., 11 (2004) 327-347. 
[17] E. Ntotsios, C. Papadimitriou, P. Panetsos, G. Karaiskos, K. Perros, P.C. Perdikaris, Bridge health monitoring system based on vibration measurements, Bulletin of Earthquake Engineering, 7 (2009) 469-483.

[18] J.L. Beck, K.V. Yuen, Model selection using response measurements: Bayesian probabilistic approach, Journal of Engineering Mechanics-Asce, 130 (2004) 192-203.

[19] M. Muto, J.L. Beck, Bayesian updating and model class selection for hysteretic structural models using stochastic simulation, Journal of Vibration and Control, 14 (2008) 7-34.

[20] C. Papadimitriou, J.L. Beck, L.S. Katafygiotis, Asymptotic expansions for reliability and moments of uncertain systems, Journal of Engineering Mechanics, 123 (1997) 1219-1229.

[21] C. Papadimitriou, J.L. Beck, L.S. Katafygiotis, Updating robust reliability using structural test data, Probabilistic Engineering Mechanics, 16 (2001) 103-113.

[22] J.L. Beck, L.S. Katafygiotis, Updating models and their uncertainties. I: Bayesian statistical framework, Journal of Engineering Mechanics-ASCE, 124 (1998) 455-461.

[23] K.-V. Yuen, Bayesian methods for structural dynamics and civil engineering, John Wiley \& Sons, 2010.

[24] J.L. Beck, Bayesian system identification based on probability logic, Structural Control \& Health Monitoring, 17 (2010) 825-847.

[25] E. Simoen, G. De Roeck, G. Lombaert, Dealing with uncertainty in model updating for damage assessment: A review, Mechanical Systems and Signal Processing, 56-57 (2015) 123-149.

[26] I. Behmanesh, B. Moaveni, G. Lombaert, C. Papadimitriou, Hierarchical Bayesian model updating for structural identification, Mechanical Systems and Signal Processing, 64-65 (2015) 360-376.

[27] E. Jaynes, Probability theory as logic, in: Maximum entropy and Bayesian methods, Springer, 1990, pp. 1-16.

[28] E.T. Jaynes, Probability theory: the logic of science, Cambridge university press, 2003.

[29] A.D. Kiureghian, O. Ditlevsen, Aleatory or epistemic? Does it matter?, Structural Safety, 31 (2009) 105-112.

[30] A. O'Hagan, J.E. Oakley, Probability is perfect, but we can't elicit it perfectly, Reliability Engineering \& System Safety, 85 (2004) 239-248.

[31] B. Goller, G. Schueller, Investigation of model uncertainties in Bayesian structural model updating, Journal of Sound and Vibration, 330 (2011) 6122-6136.

[32] B. Peeters, G. De Roeck, One-year monitoring of the Z 24-Bridge: environmental effects versus damage events, Earthquake Engineering \& Structural Dynamics, 30 (2001) 149-171.

[33] A.M. Yan, G. Kerschen, P. De Boe, J.C. Golinval, Structural damage diagnosis under varying environmental conditions - Part I: A linear analysis, Mechanical Systems and Signal Processing, 19 (2005) 847-864.

[34] A.M. Yan, G. Kerschen, P. De Boe, J.C. Golinval, Structural damage diagnosis under varying environmental conditions - part II: local PCA for non-linear cases, Mechanical Systems and Signal Processing, 19 (2005) 865-880.

[35] W.H. Hu, C. Moutinho, E. Caetano, F. Magalhaes, A. Cunha, Continuous dynamic monitoring of a lively footbridge for serviceability assessment and damage detection, Mechanical Systems and Signal Processing, 33 (2012) 38-55. 
[36] D.F. Giraldo, S.J. Dyke, J.M. Caicedo, Damage detection accommodating varying environmental conditions, Structural Health Monitoring-an International Journal, 5 (2006) 155-172.

[37] M.T. Yarnold, F.L. Moon, A. Emin Aktan, Temperature-Based Structural Identification of Long-Span Bridges, Journal of Structural Engineering, (2015).

[38] K.-V. Yuen, S.-C. Kuok, Modeling of environmental influence in structural health assessment for reinforced concrete buildings, Earthq. Eng. Eng. Vib., 9 (2010) 295-306.

[39] P. Moser, B. Moaveni, Environmental effects on the identified natural frequencies of the Dowling Hall Footbridge, Mechanical Systems and Signal Processing, 25 (2011) 2336-2357.

[40] F. Magalhães, A. Cunha, E. Caetano, Vibration based structural health monitoring of an arch bridge: from automated OMA to damage detection, Mech. Syst. Signal Proc., 28 (2012) 212-228.

[41] B. Moaveni, I. Behmanesh, Effects of changing ambient temperature on finite element model updating of the Dowling Hall Footbridge, Engineering Structures, 43 (2012) 58-68.

[42] E.J. Cross, K.Y. Koo, J.M.W. Brownjohn, K. Worden, Long-term monitoring and data analysis of the Tamar Bridge, Mechanical Systems and Signal Processing, 35 (2013) 16-34.

[43] P. Moser, B. Moaveni, Design and deployment of a continuous monitoring system for the Dowling Hall Footbridge, Experimental Techniques, 37 (2013) 15-26.

[44] B. Peeters, G. De Roeck, Reference-based stochastic subspace identification for output-only modal analysis, Mechanical systems and signal processing, 13 (1999) 855-878.

[45] I. Behmanesh, B. Moaveni, Probabilistic identification of simulated damage on the Dowling Hall footbridge through Bayesian finite element model updating, Struct. Control. Health Monit., 22 (2015) 463-483.

[46] CSI, Integrated Finite Element Analysis and Design of Structures Basic Analysis Reference Manual, Berkeley, CA, USA, 2015.

[47] MathWorks, MATLAB User's Guide, MathWorks Inc., Natick, MA, 2014.

[48] F.C. Filippou, M.F. Constantinides, Getting started guide and simulation examples, in, Technical report NEESgrid-2004-22. Berkeley (CA): Civil and Environmental Eng. Dept. University of California at Berkeley, 2004.

[49] S. Geman, D. Geman, Stochastic relaxation, Gibbs distributions, and the Bayesian restoration of images, Pattern Analysis and Machine Intelligence, IEEE Transactions on, (1984) 721-741.

[50] A.E. Gelfand, Sampling-Based Approaches to Calculating Marginal Densities, Journal of the American Statistical Association, 85 (1990) 398-409.

[51] L.S. Katafygiotis, J.L. Beck, Updating models and their uncertainties. II: Model identifiability, Journal of Engineering Mechanics-Asce, 124 (1998) 463-467.

[52] J.Y. Ching, Y.C. Chen, Transitional markov chain monte carlo method for Bayesian model updating, model class selection, and model averaging, JOURNAL OF ENGINEERING MECHANICS-ASCE, 133 (2007) 816-832.

[53] P.E. Hadjidoukas, P. Angelikopoulos, C. Papadimitriou, P. Koumoutsakos, П4U: A high performance computing framework for Bayesian uncertainty quantification of complex models, Journal of Computational Physics, 284 (2015) 1-21. 
[54] I. Behmanesh, S. Yousefianmoghadam, A. Nozari, B. Moaveni, A. Stavridis, Effects of Prediction Error Bias on Model Calibration and Response Prediction of a 10-Story Building in: 34th International Modal Analysis Conference, Springer, Orlando, FL, USA, 2016.

[55] C. Andrieu, J. Thoms, A tutorial on adaptive MCMC, Statistics and Computing, 18 (2008) 343-373.

[56] P. Angelikopoulos, C. Papadimitriou, P. Koumoutsakos, Bayesian uncertainty quantification and propagation in molecular dynamics simulations: a high performance computing framework, The Journal of chemical physics, 137 (2012) 144103.

[57] W.R. Gilks, S. Richardson, D.J. Spiegelhalter, Markov chain Monte Carlo in practice, Chapman \& Hall, Boca Raton, FL, 1998.

[58] R. Fox, M. Kapoor, Rates of change of eigenvalues and eigenvectors, AIAA journal, 6 (1968) 2426-2429.

[59] I. Behmanesh, B. Moaveni, Bayesian FE Model Updating in the Presence of Modeling Errors, in: Model Validation and Uncertainty Quantification, Volume 3, Springer, 2014, pp. 119-133.

[60] A. Gelman, Prior distributions for variance parameters in hierarchical models (comment on article by Browne and Draper), Bayesian analysis, 1 (2006) 515-534.

[61] K. Christodoulou, C. Papadimitriou, Structural identification based on optimally weighted modal residuals, Mechanical Systems and Signal Processing, 21 (2007) 4-23.

[62] B. Goller, J.L. Beck, G.I. Schueller, Evidence-Based Identification of Weighting Factors in Bayesian Model Updating Using Modal Data, Journal of Engineering Mechanics-ASCE, 138 (2012) 430-440.

[63] I. Behmanesh, B. Moaveni, Probabilistic Damage Identification of the Dowling Hall Footbridge through Hierarchical Bayesian Model updating, in: 12th International Conference on Applications of Statistics and Probability in Civil Engineering (ICASP12), Vancouver, Canada, 2015.

[64] D. Draper, Assessment and propagation of model uncertainty, Journal of the Royal Statistical Society. Series B (Methodological), (1995) 45-97.

[65] J.A. Hoeting, D. Madigan, A.E. Raftery, C.T. Volinsky, Bayesian model averaging: A tutorial, Statistical Science, 14 (1999) 382-401. 

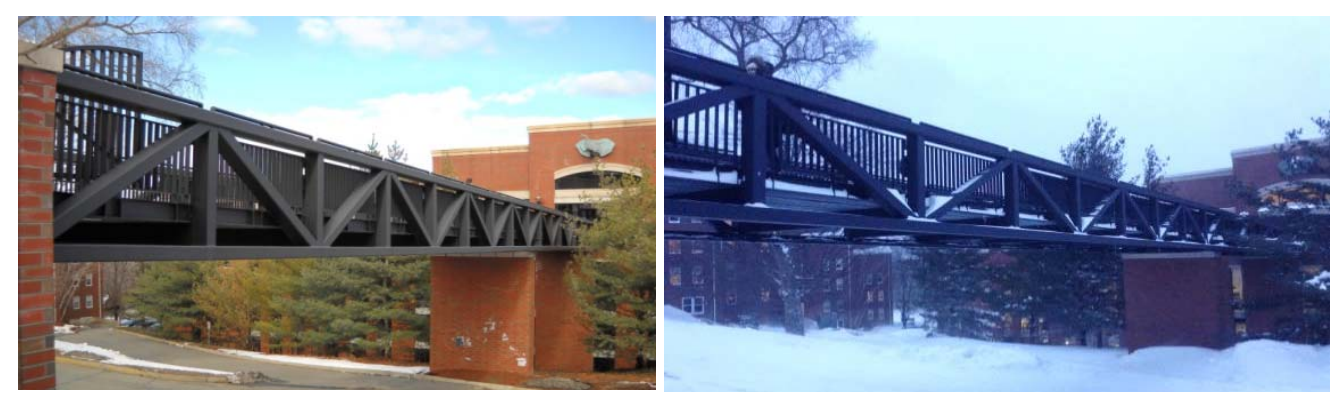

Fig. 1 South view of Dowling Hall Footbridge

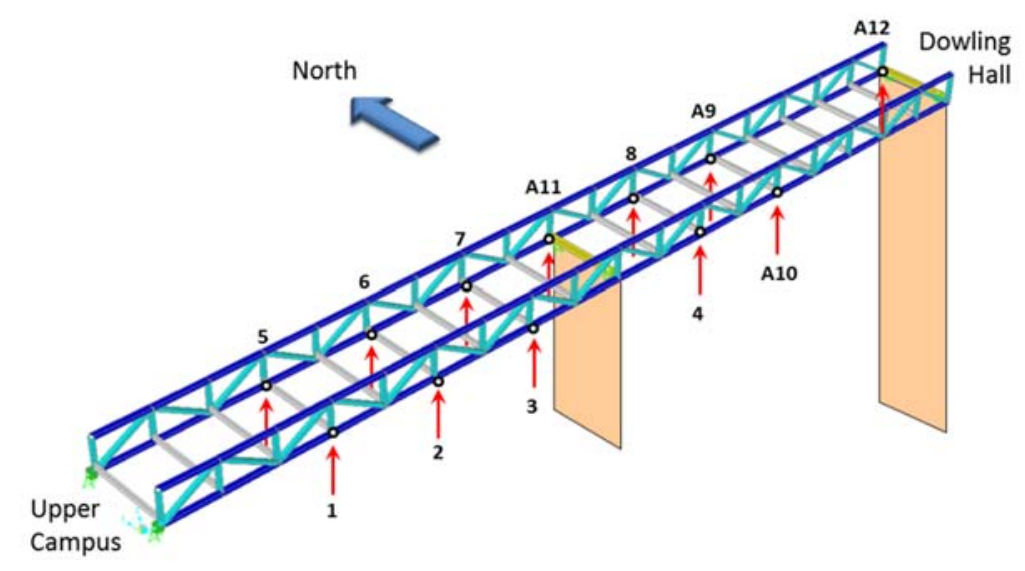

Fig. 2 Layout of accelerometers on the bridge 

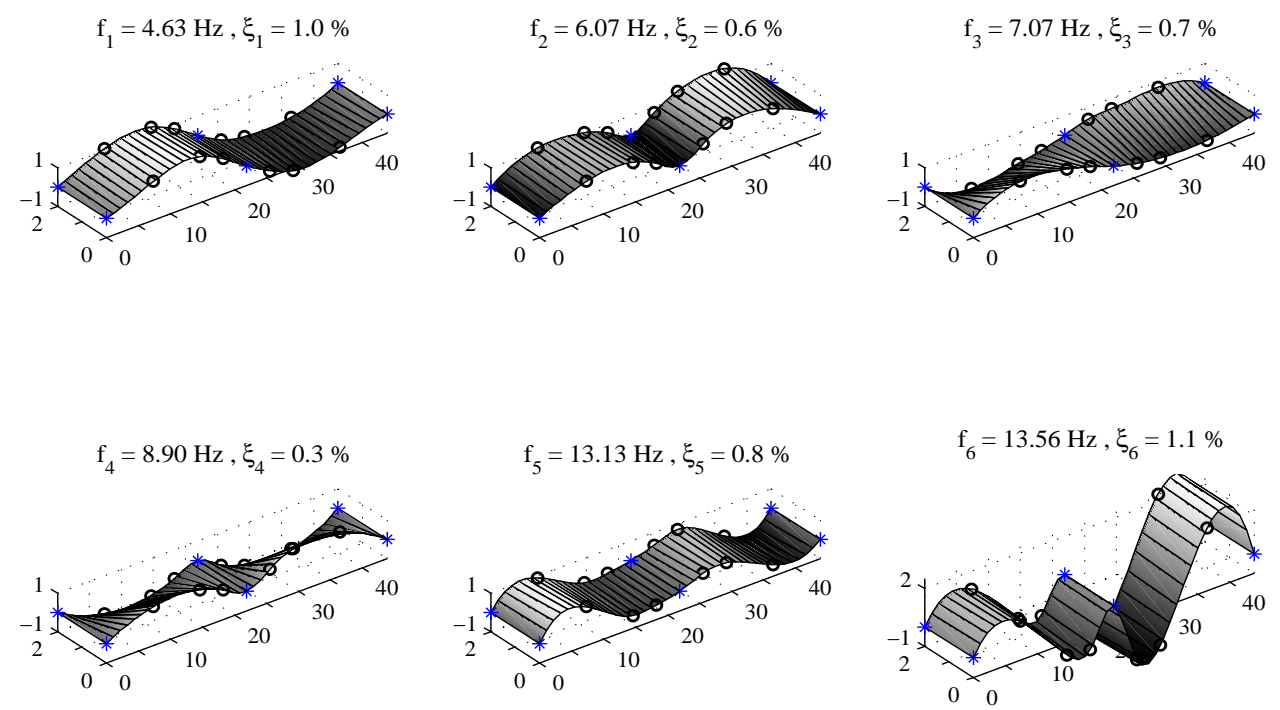

Fig. 3 The six vibration modes of the footbridge previously reported in [39, 41, 45]
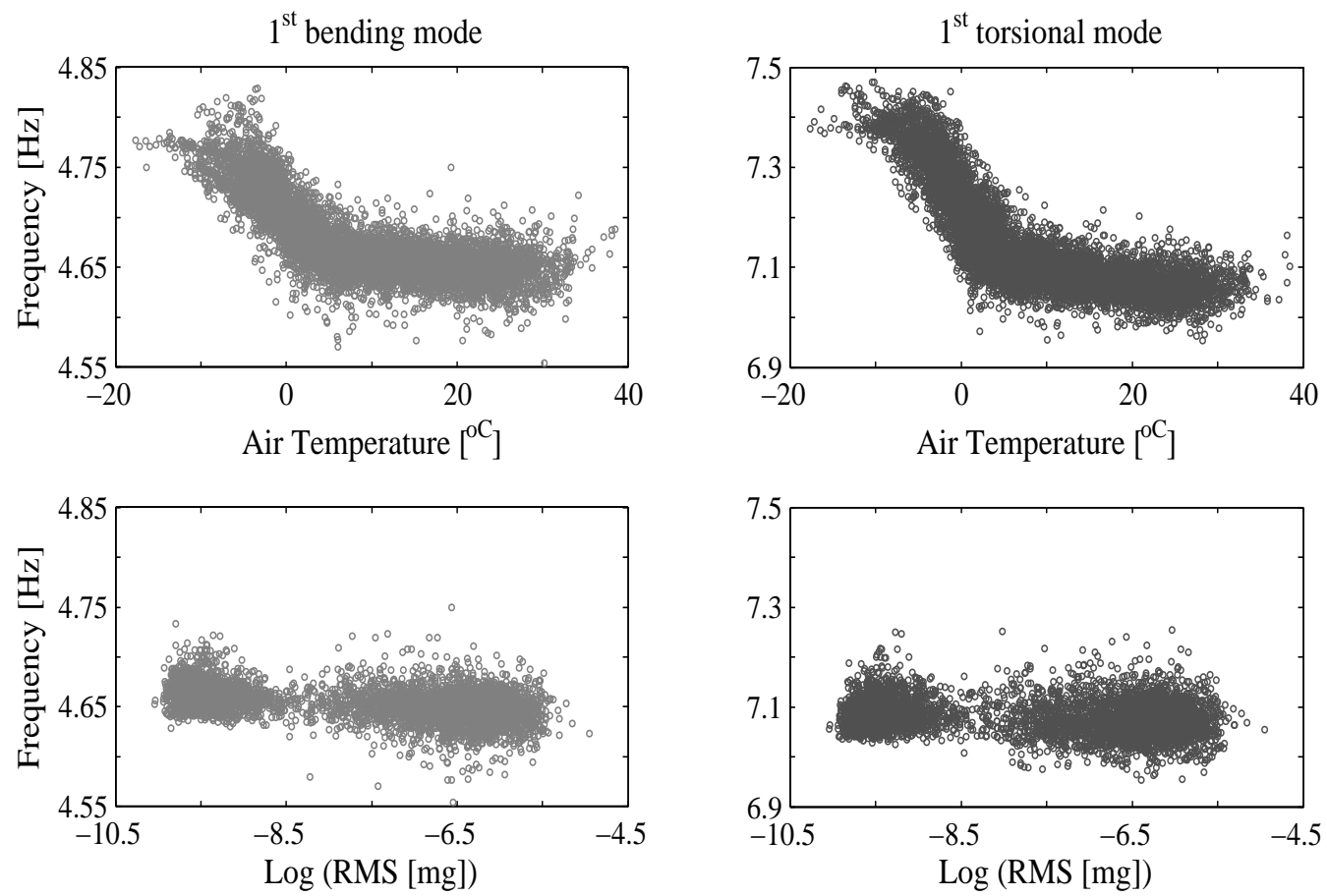

Fig. 4 Effects of ambient temperature and excitation amplitude on the identified natural frequencies 


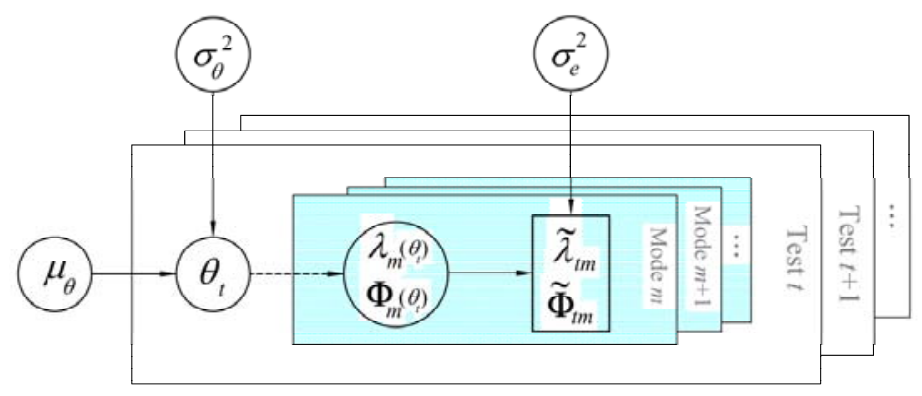

Fig. 5 Graphical representation of the probabilistic updating framework at information level 1
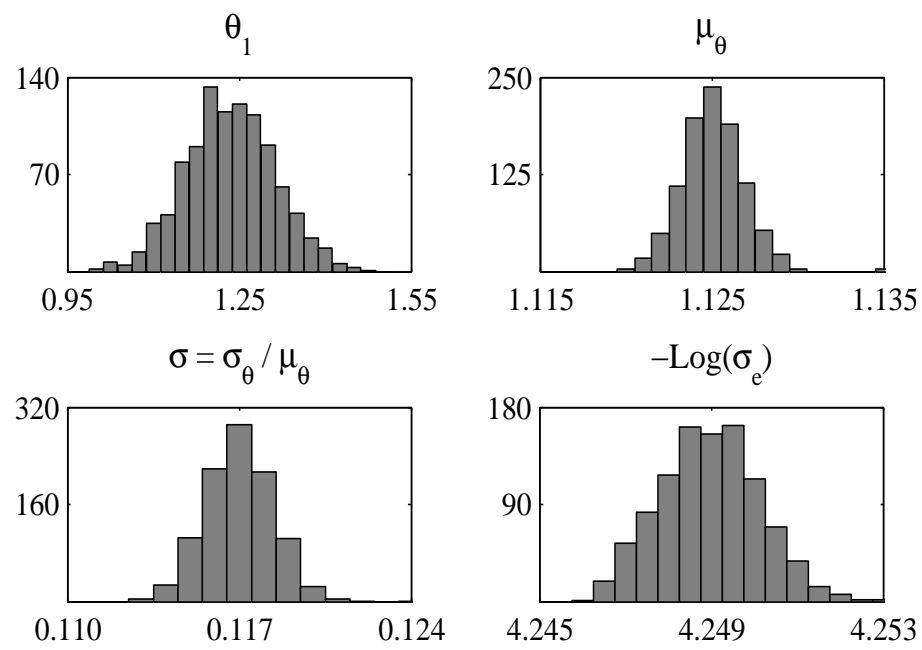

Fig. 6 Histogram of $\theta_{1}, \mu_{\theta}, \sigma$ and $\log \left(\sigma_{e}\right)$ from the generated Gibbs Samples at information level 1 


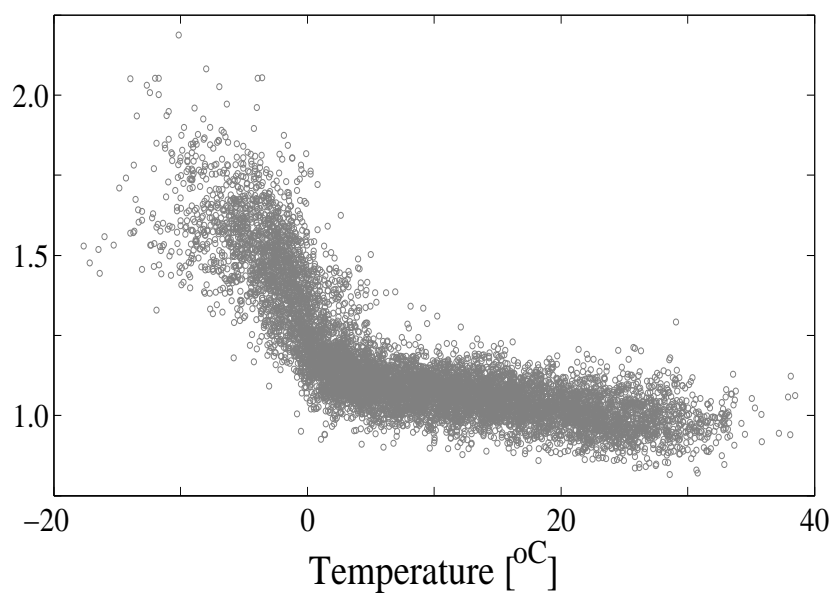

Fig. 7 Optimum structural parameters $\hat{\theta}_{t}$ versus air temperature
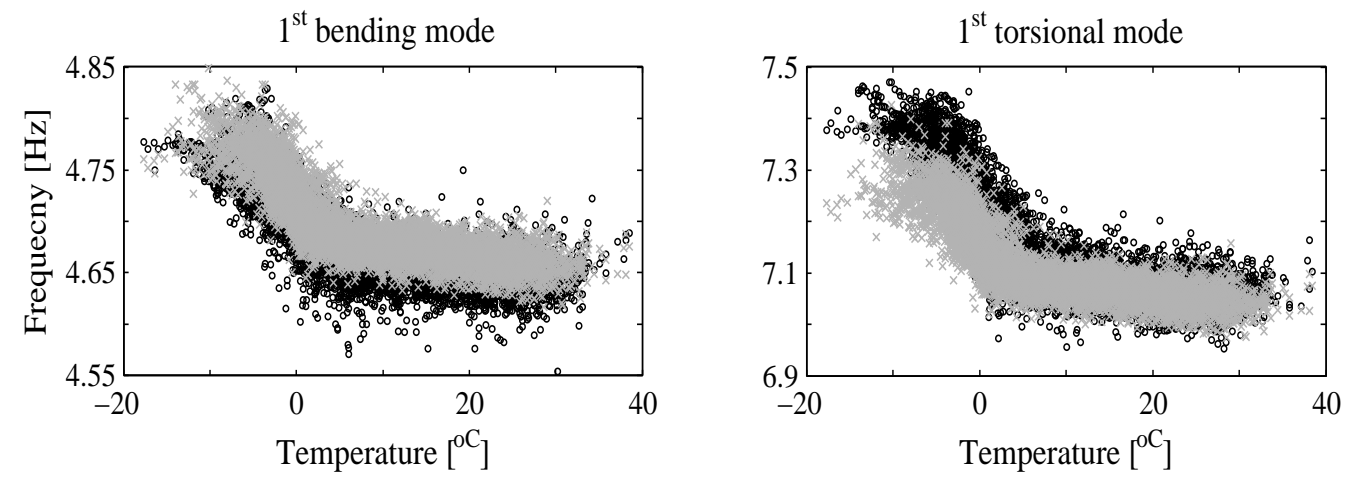

Fig. 8 Identified (black circles) versus model-calculated natural frequencies (gray crosses) 

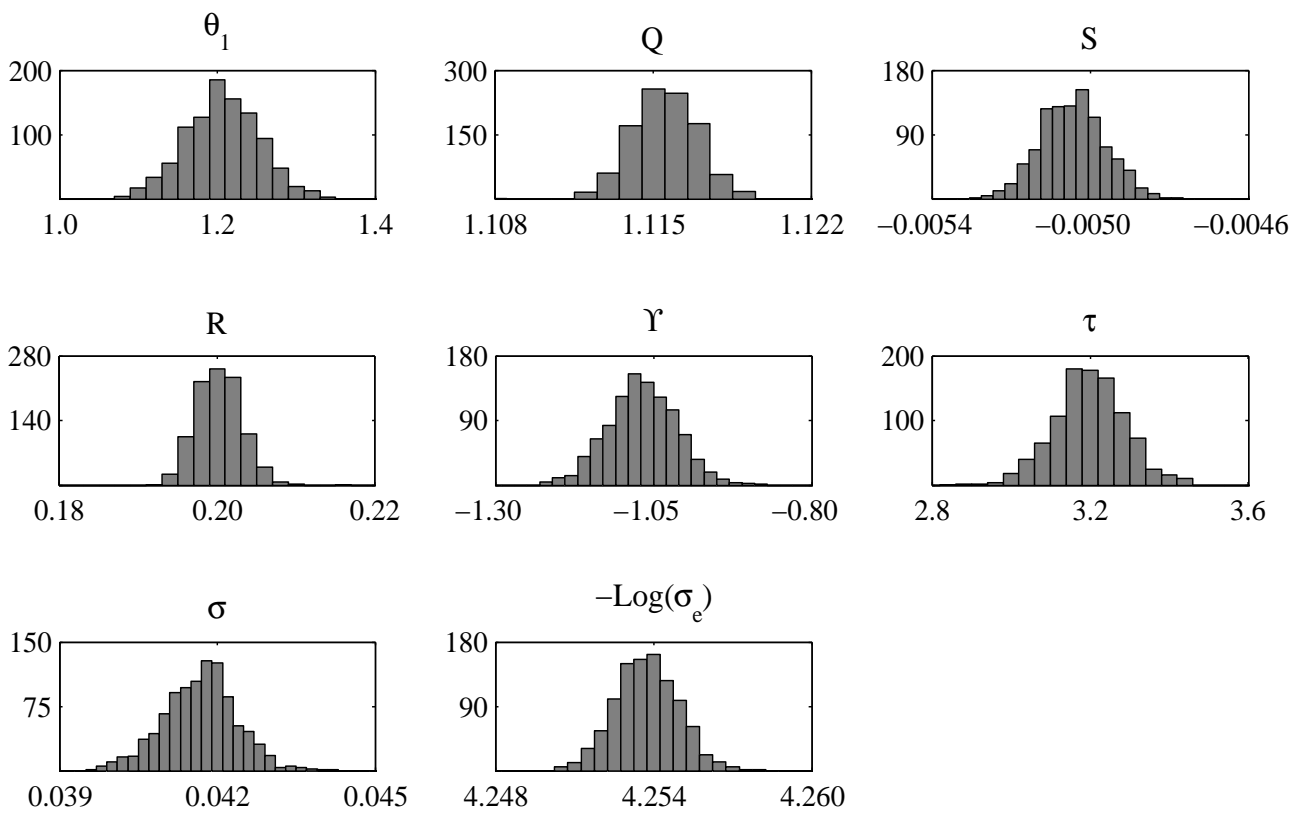

Fig. 9 Histogram of $\theta_{l}$ and 7 hyper-parameters from the generated Gibbs Samples at information level 2

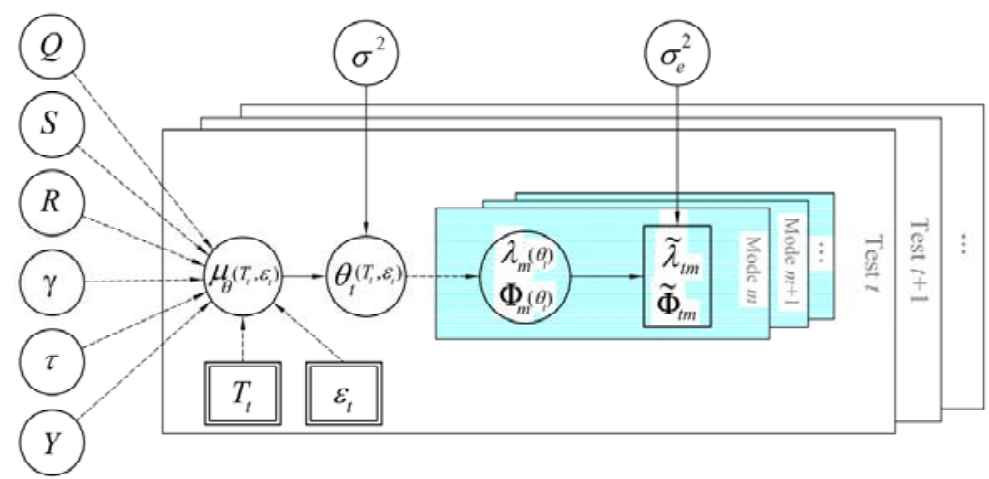

Fig. 10 Graphical representation of the probabilistic updating framework at information level 3 

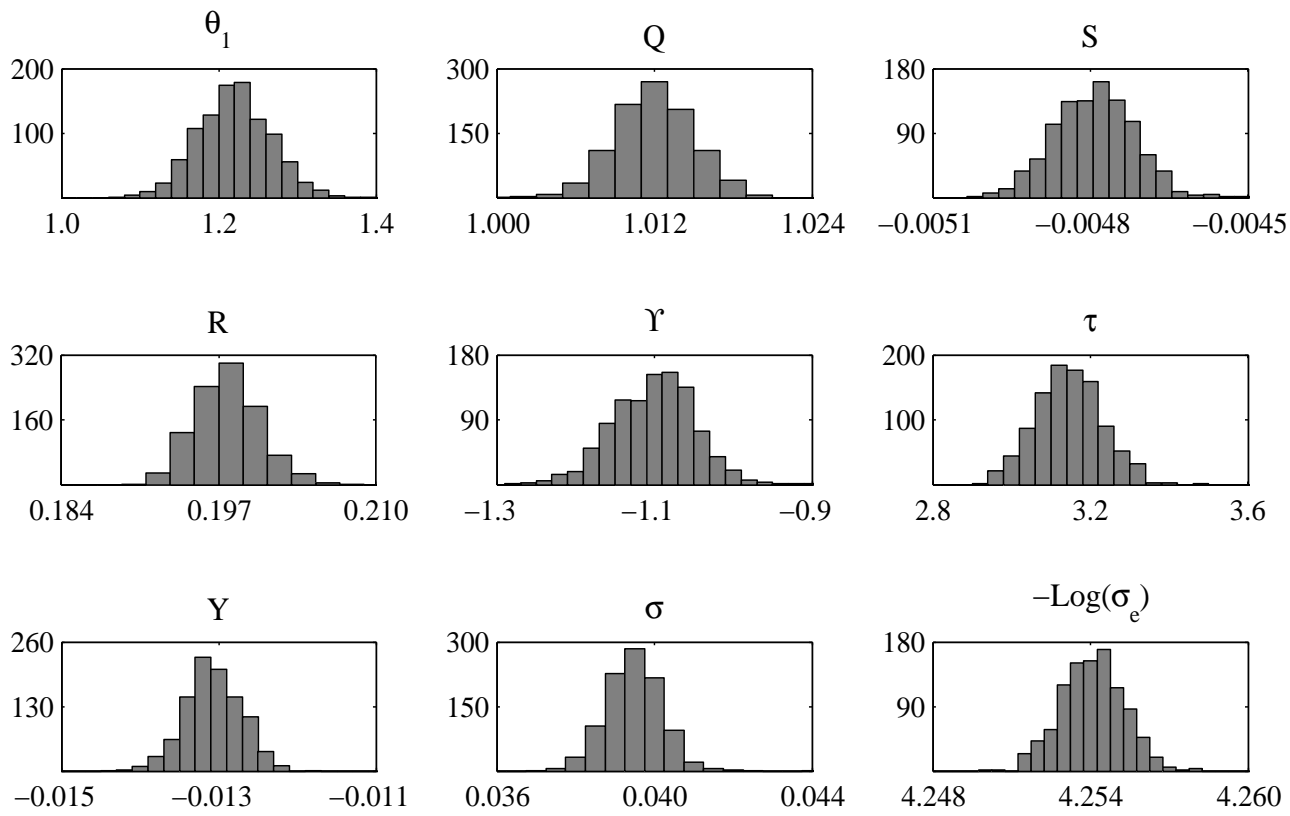

Fig. 11 Histogram of $\theta_{1}$ and hyper-parameters from the Gibbs Samples at information level 3
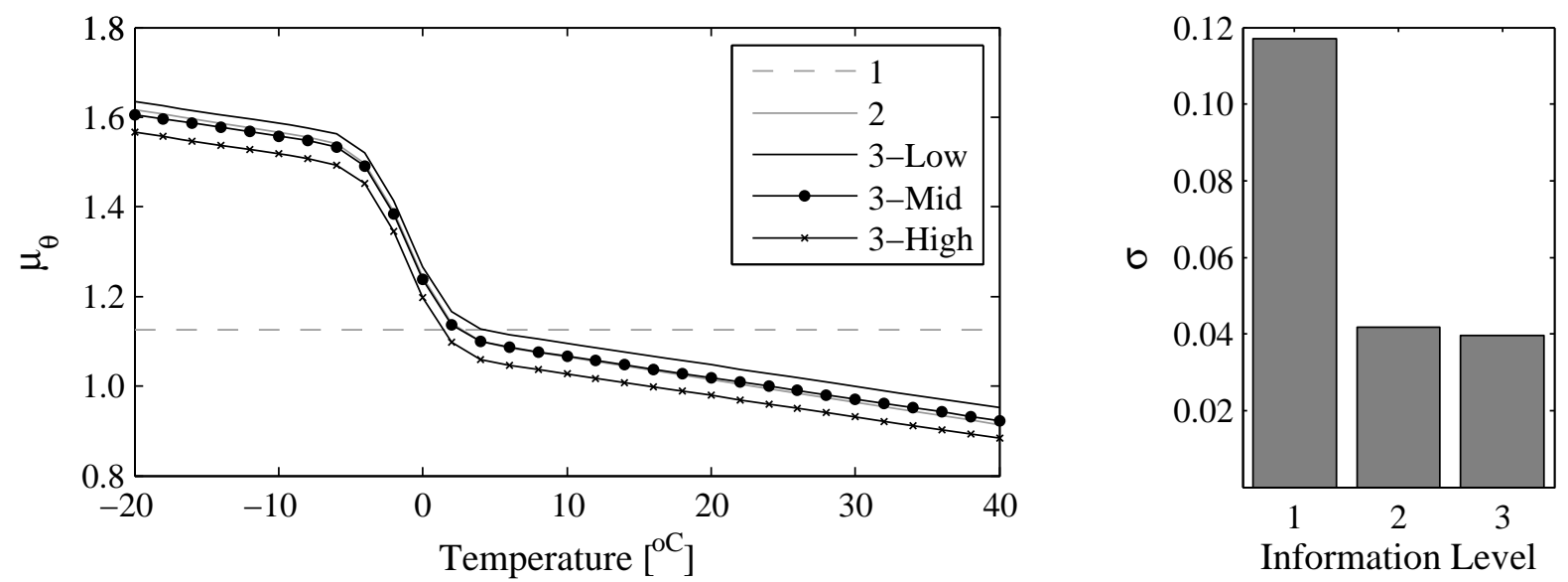

Fig. 12 Mean and coefficient-of-variation of $\theta$ estimated at different information levels 


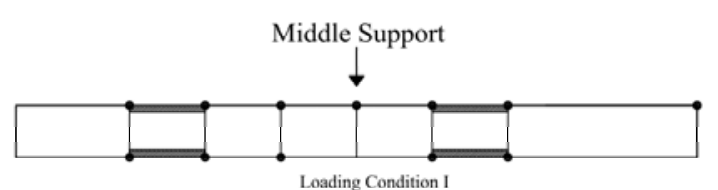

- Accelerometer Location

Location of Added Mass

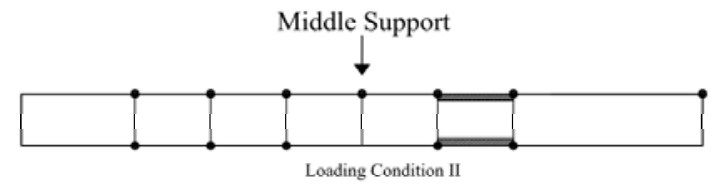

- Accelerometer Location

Location of Added Mass

Fig. 13 Loading scenario I (left) and loading scenario II (right)
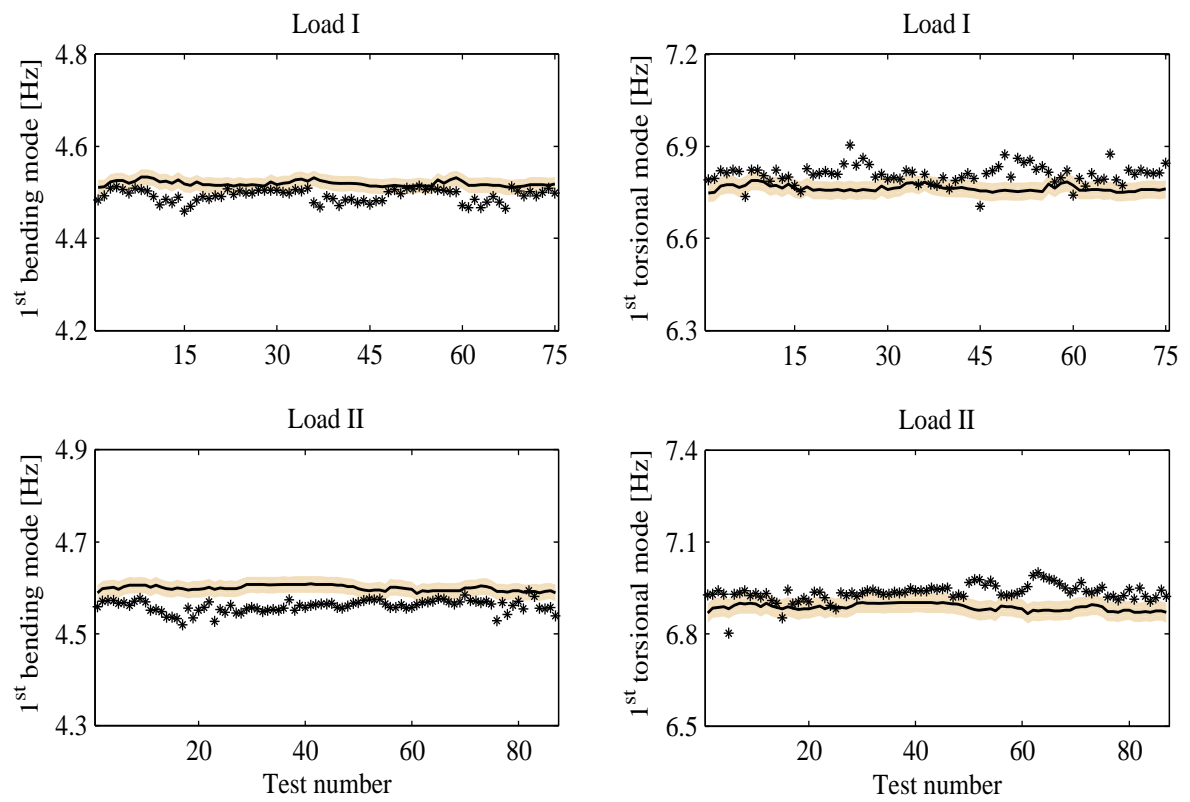

Fig. 14 Model predicted and identified natural frequencies at the two loading scenario without accounting for modeling error uncertainties 

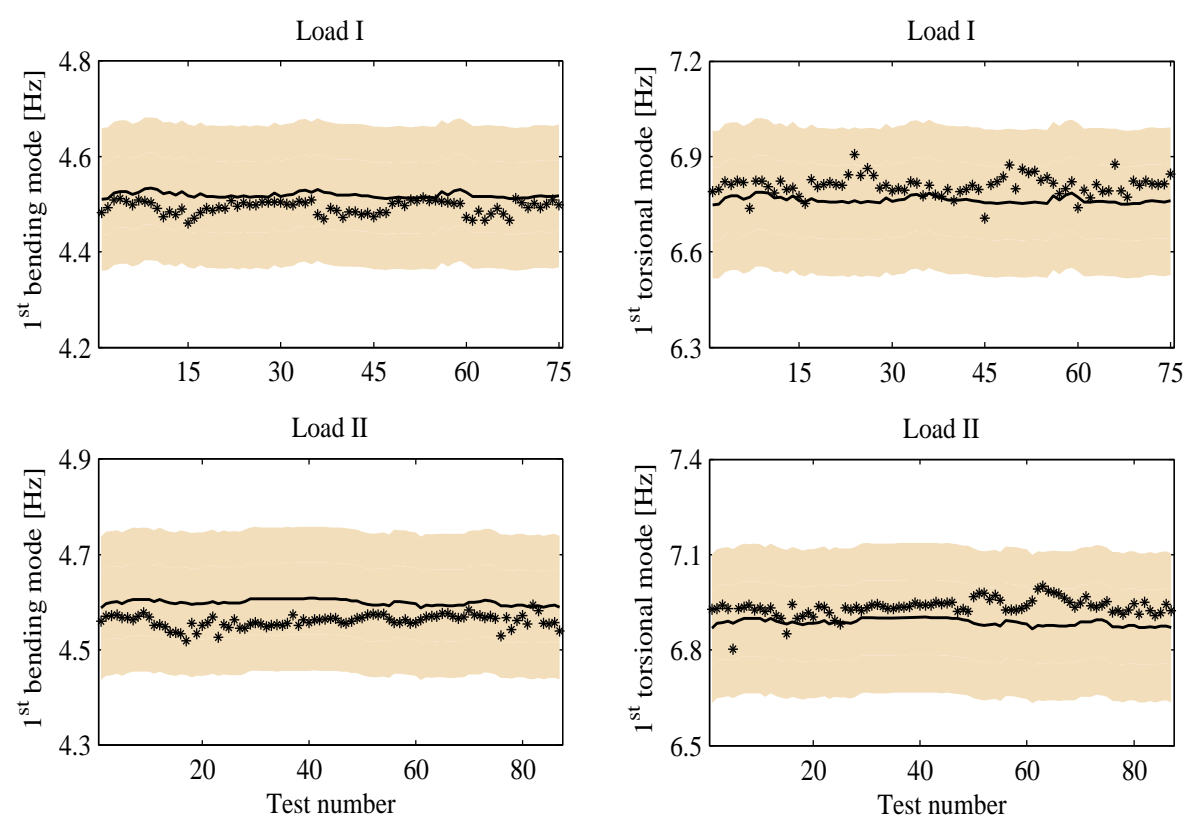

Fig. 15 Model predicted and identified natural frequencies at the two loading conditions with propagation of modeling error uncertainties

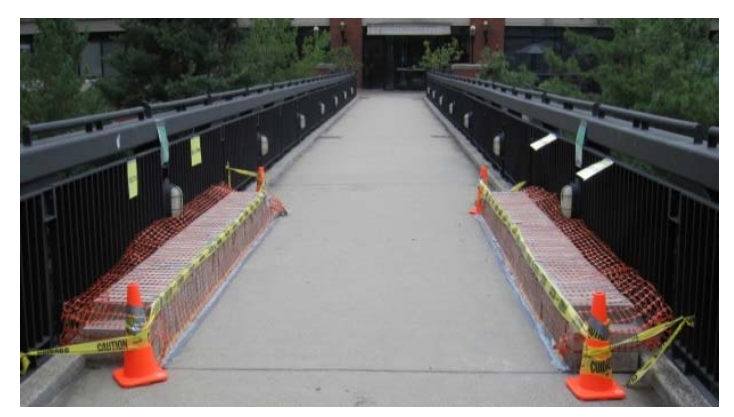

Fig. 16 Added mass on footbridge deck 

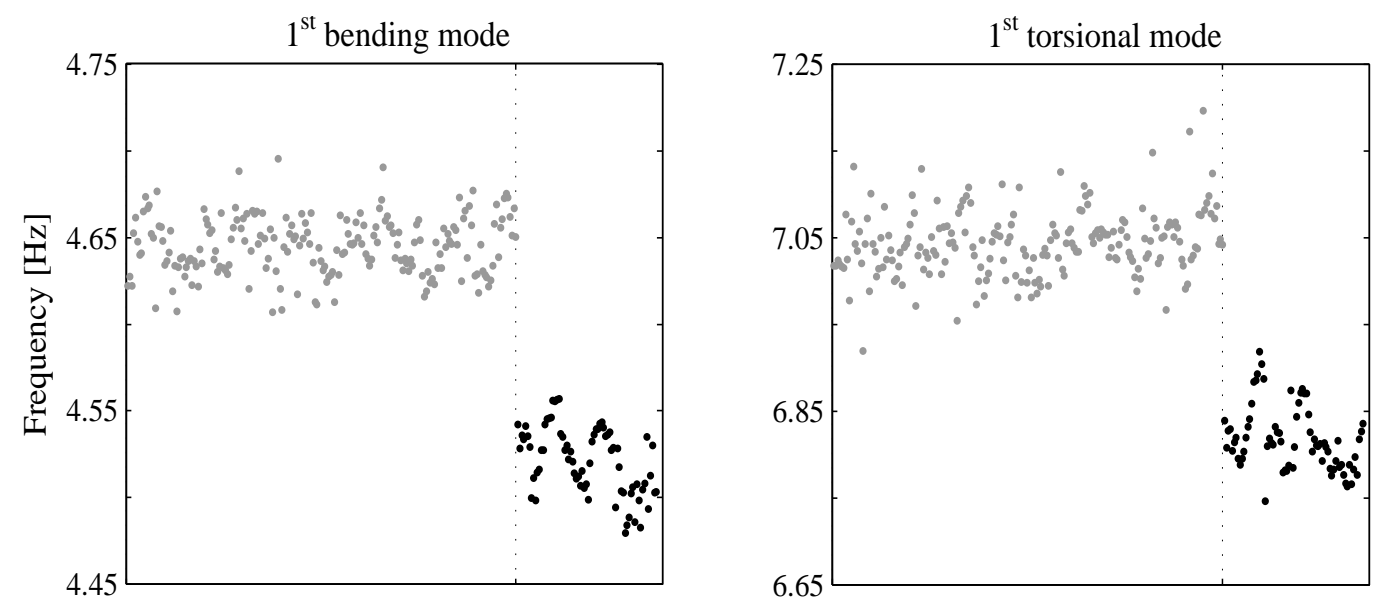

Fig. 17 Natural frequencies in the damaged and undamaged states

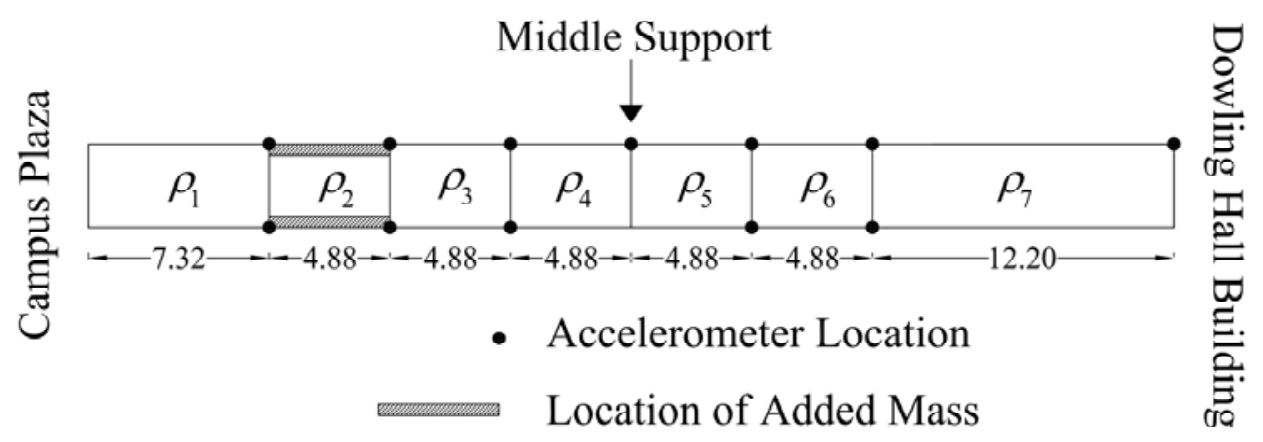

Fig. 18 Considered updating structural mass parameters for damage identification 


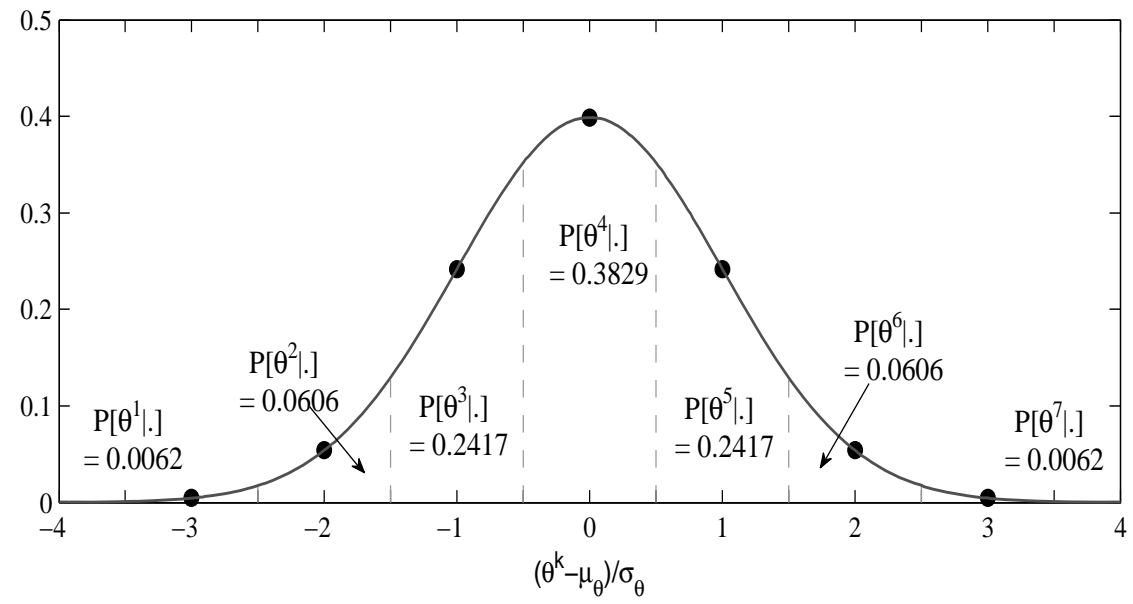

Fig. 19 Discretization of parameter $\theta$ and its probability mass function

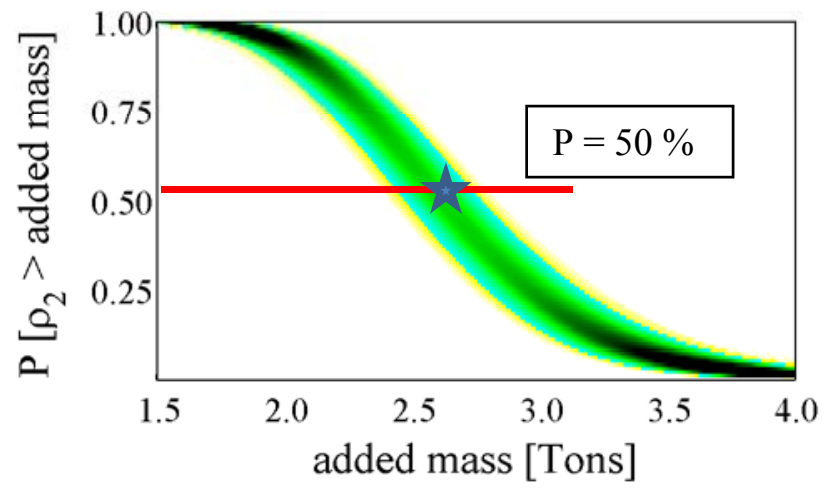

Fig. 20 Probability of damage (added mass) exceedance on segment 2 

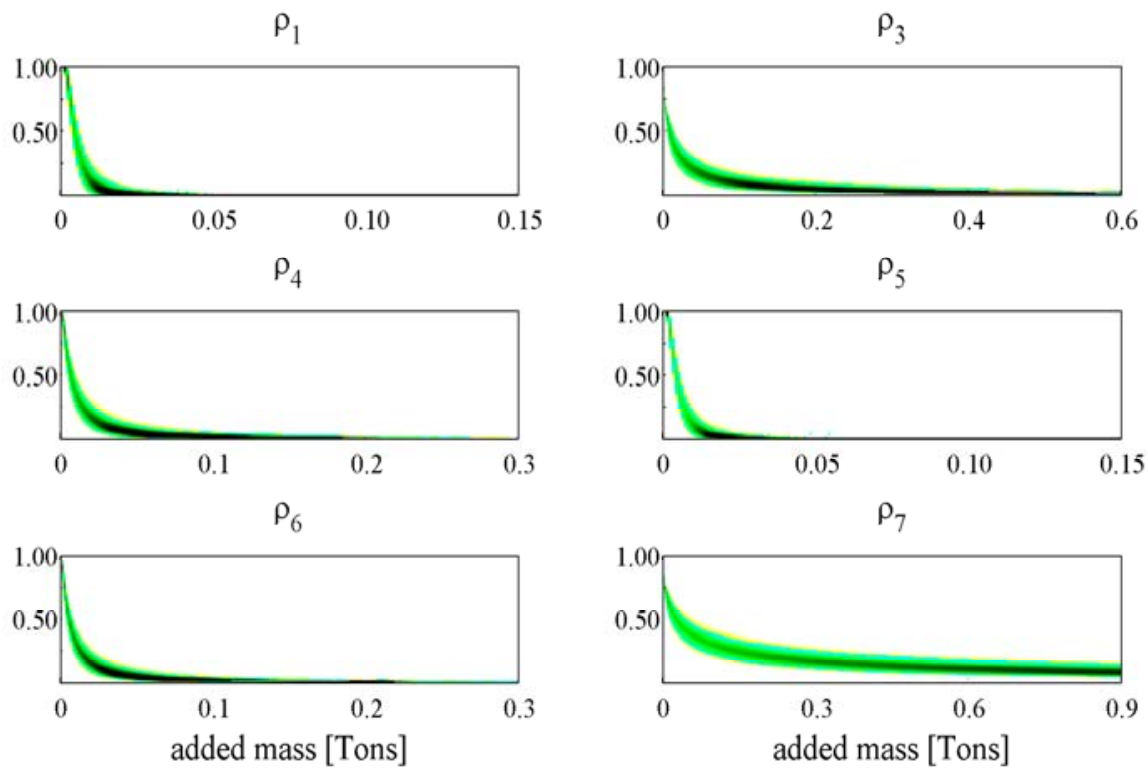

Fig. 21 Probability of damage (added mass) exceedance on the unloaded segments 